\title{
Intrinsic operators for the translationally-invariant many-body problem
}

\author{
Mark A. Caprio, ${ }^{1}$ Anna E. McCoy, ${ }^{1,2}$ and Patrick J. Fasano ${ }^{1}$ \\ ${ }^{1}$ Department of Physics, University of Notre Dame, \\ Notre Dame, Indiana 46556-5670, USA \\ ${ }^{2}$ TRIUMF, Vancouver, British Columbia V6T 2A3, Canada
}

(Dated: August 20, 2020)

\begin{abstract}
The need to enforce fermionic antisymmetry in the nuclear many-body problem commonly requires use of single-particle coordinates, defined relative to some fixed origin. To obtain physical operators which nonetheless act on the nuclear many-body system in a Galilean-invariant fashion, thereby avoiding spurious center-of-mass contributions to observables, it is necessary to express these operators with respect to the translational intrinsic frame. Several commonly-encountered operators in nuclear many-body calculations, including the magnetic dipole and electric quadrupole operators (in the impulse approximation), and generators of $U(3)$ and $\operatorname{Sp}(3, \mathbb{R})$ symmetry groups, are bilinear in the coordinates and momenta of the nucleons and, when expressed in intrinsic form, become two-body operators. To work with such operators in a second-quantized many-body calculation, it is necessary to relate three distinct forms: the defining intrinsic-frame expression, an explicitly two-body expression in terms of two-particle relative coordinates, and a decomposition into one-body and separable two-body parts. We establish the relations between these forms, for general (non-scalar and non-isoscalar) operators bilinear in coordinates and momenta.
\end{abstract}

Keywords: Galilean-invariant intrinsic operators, nuclear many-body problem, electromagnetic observables, center-of-mass contamination, no-core configuration interaction (NCCI) calculations, no-core shell model (NCSM), nuclear $\mathrm{SU}(3)$ and $\mathrm{Sp}(3, \mathbb{R})$ symmetries

\section{INTRODUCTION}

In studying the nuclear system, the states of interest are those involving excitation of the intrinsic structure of the nucleus in its comoving inertial reference frame, not those involving "spurious" motion of the center of mass. The nuclear many-body problem is translationally invariant. Except for the contribution to the kinetic energy operator arising from center-ofmass motion, the problem is, moreover, Galilean invariant, i.e., also invariant under boosts to the momenta. Therefore, if the nuclear problem could be solved in the full, untruncated many-body space, then the spectrum of nuclear excited states would contain an intrinsic spectrum, reflecting intrinsic excitations of the nucleons relative to each other. Onto this intrinsic spectrum would be superposed an infinite continuum of copies, each representing the combination of this intrinsic structure with a different center-of-mass motion.

In practical calculations, there is a fundamental conflict between choosing a computational framework which manifestly reflects the Galilean invariant nature of the Hamiltonian and one which readily respects the antisymmetry constraints imposed by the fermionic statistics of the nucleons. A natural starting point for the translationally invariant problem is provided by a coordinate system, such as Jacobi coordinates, which explicitly separates the center-of-mass coordinate from the other, intrinsic coordinates [82, 83]. However, defining 
a many-body basis in terms of the intrinsic coordinates is challenging. In particular, it becomes prohibitively difficult to impose antisymmetry in Jacobi coordinates [84] as the number of particles increases.

Antisymmetry is more easily enforced by representing the system in terms of antisymmetrized products (Slater determinants) of single-particle states. These single-particle states are defined with respect to a common, fixed origin, that is, with respect to laboratory-frame coordinates. Thus, the many-body problem is typically solved in a framework which does not manifestly preserve translational invariance.

Even so, it is possible to compute observables as they would be measured in the intrinsic frame, insensitive to the center-of-mass motion. To do so, we must ensure that we calculate observables using suitable Galilean-invariant intrinsic operators, which reduce to the operator of interest when evaluated in the intrinsic frame [85 89].

Many operators of interest for the nuclear many-body problem involve angular-momentum coupled products of the form $(\mathbf{x} \times \mathbf{x}),(\mathbf{x} \times \mathbf{p})$, and $(\mathbf{p} \times \mathbf{p})$, that is, bilinear in coordinates and/or momenta. Although these operators are one-body operators when expressed in the laboratory frame, they become two-body operators when realized as intrinsic operators. To work with the Galilean-invariant intrinsic operators obtained from such bilinear expressions, in a second-quantized many-body calculation, we therefore need to evaluate their two-body matrix elements. To do so, it is necessary to relate three distinct representations of these operators: (1) the defining intrinsic-frame expression, (2) an explicitly two-body expression in terms of two-particle relative coordinates, and (3) a decomposition into one-body and separable two-body parts.

In the case of the rotational scalar intrinsic kinetic energy operator, commonly used in nuclear configuration interaction calculations, the relations among these forms are long familiar to shell model practitioners [90, 91. The structure of the intrinsic squared radius operator [92 94], which enters into nuclear charge radius calculations (and furthermore serves as the leading-order electric monopole operator), is essentially identical to that of the kinetic energy, but with the introduction of charge or isosopin dependence. Among the highermultipolarity electromagnetic transition operators [85, 88, in the impulse approximation, the magnetic dipole and electric quadrupole operators are likewise bilinear in coordinates and/or momenta, though no longer scalar operators like those just mentioned.

Bilinear operators, beyond representing physical observables, serve to define the symmetry structure of the nuclear many-body problem. The full set of bilinears provide the generators of the symplectic group $\operatorname{Sp}(3, \mathbb{R})$ in three dimensions [95-97]. This group is closely linked to the dynamics of the many-body problem in three-dimensional space and contains Elliott's U(3) group [98, 99] as a subgroup. The intrinsic forms of all these operators share a common structure.

Here we derive systematic extensions of the relations for intrinsic operators, from the familiar rotational scalar and isoscalar case, to the general case of rotational nonscalar and isovector bilinear operators. These results are motivated for use in multiple contexts, including in calculating intrinsic observables in the $a b$ initio no-core configuration interaction (NCCI) [or no-core shell model (NCSM)] approach [100] and in establishing the calculational machinery for the symplectic no-core configuration interaction (SpNCCI) framework [101. 103. While the derivations are straightforward, in principle, careful attention must be paid to the various phase and normalization factors which arise if the results are to be practically useful in nuclear many-body calculations. These include $A$-dependent (or $N$ and $Z$-dependent) counting factors, as well as insidious factors of 2 which can easily be 
overlooked by the unwary when relating operators on the two-body relative system and the full $A$-body system.

The basic ideas and approaches developed here may be applied further to operators defined in terms of higher-order products of the coordinates and/or momenta. For instance, the electric multipole operator of rank $\lambda$ may be expressed as an angular-momentum coupled product $(\mathbf{x} \times \mathbf{x} \times \cdots \times \mathbf{x})$ of order $\lambda$ in the coordinates. Although this operator is a one-body operator when expressed in the laboratory frame, the corresponding intrinsic operator is a $\lambda$-body operator.

For motivation and context, we first provide some elaboration of the ways in which the relations considered here enter into both traditional and symmetry-adapted NCCI nuclear many-body calculations (Sec. II). There are ample opportunities for ambiguity arising from alternative conventions for translating between relative and single-particle coordinates. We therefore next carefully set out notation and definitions for one-body and two-body operators, relative and center-of-mass coordinates for the two-body system, and intrinsic coordinates for the many-body system (Sec. III).

Before addressing the bilinear intrinsic operators, it is instructive to first consider the electric dipole operator as an intrinsic operator (Sec. IV). Although this operator is simply linear (not bilinear) in coordinates, it provides a more transparent context in which to establish the approaches required for the bilinear operators.

We finally turn to the generic nonscalar bilinear operator, in both its isoscalar and isovector variants. We lay out how such an operator is represented as a two-body intrinsic operator, and then how it may be represented in terms of either separable or relative two-body operators (Sec. VD). Preliminary results were presented in Ref. [101].

In appendices, we provide additional, more specific results for practical reference in working with two-body, bilinear intrinsic operators: We review the explicit expressions for several physically relevant operators, cast in the generic bilinear form (Appendix A). We account for the effect of the proton-neutron mass difference on the intrinsic kinetic energy operator (Appendix B). We note the summation identities needed in converting between one-body and two-body forms of operators (Appendix C). We give expressions casting the one-body spin operators as two-body operators, so that they can be included in calculations of twobody matrix elements for the intrinsic magnetic dipole operator (Appendix D). We obtain relations for evaluating isospin-reduced matrix elements of the isovector forms of the twobody intrinsic operators (Appendix E). Then, to facilitate work with the harmonic oscillator creation and annihilation (ladder) operators, we review definitions and relations for the harmonic oscillator ladder operators on the single-particle, relative, and intrinsic degrees of freedom (Appendix F).

\section{BACKGROUND: INTRINSIC OPERATORS IN THE NUCLEAR MANY-BODY PROBLEM}

The Galilean-invariant intrinsic form, which does not introduce center-of-mass contamination, is obtained, for an operator expressed in coordinates $\mathbf{x}$ and/or momenta $\mathbf{p}$, by the substitutions $\mathbf{x}_{i} \rightarrow \mathbf{x}_{i}^{\prime}$ and $\mathbf{p}_{i} \rightarrow \mathbf{p}_{i}^{\prime}$. The Galilean-invariant intrinsic coordinates $\mathbf{x}_{i}^{\prime}$ and momenta $\mathbf{p}_{i}^{\prime}$ are defined relative to the center of mass and center of momentum, respectively. As context for the results for general bilinear operators obtained below, we review some essential observations for the intrinsic kinetic energy [90, 91, 104] and squared radius operators (Sec. II A), then comment on the implications of the existence of intrinsic $\operatorname{Sp}(3, \mathbb{R}) \supset \mathrm{U}(3)$ 
group generators for the structure of the space used in many-body calculations (Sec. II B). The special properties of the $N_{\max }$ truncation [100] for NCCI calculations follow as a special case.

\section{A. Kinetic energy and observables}

The "naive" physical Hamiltonian for the nuclear many-body system, $H=T+V$, as obtained in the laboratory frame, involves the total kinetic energy $T=\left(2 m_{N}\right)^{-1} \sum_{i} \mathbf{p}_{i} \cdot \mathbf{p}_{i}$ of the nucleons. ${ }^{1}$ While the many-body interaction operator $V$ is Galilean invariant, the kinetic energy operator violates Galilean invariance, in that the total (center-of-mass) momentum of the system enters into the kinetic energy. The full kinetic energy is thus not invariant under Galilean (momentum) boosts.

Galilean invariance is recovered by substituting the intrinsic momenta $\mathbf{p}_{i}^{\prime}$ for the momenta $\mathbf{p}_{i}$, giving the intrinsic kinetic energy

$$
T^{\prime}=\frac{1}{2 m_{N}} \sum_{i}\left(\mathbf{p}_{i}-\frac{1}{A} \sum_{j} \mathbf{p}_{j}\right) \cdot\left(\mathbf{p}_{i}-\frac{1}{A} \sum_{k} \mathbf{p}_{k}\right) .
$$

Several useful observations and relations may be obtained, more or less directly, from this expression.

First, the kinetic energy then separates as $T=T^{\prime}+T_{\mathrm{cm}}$, into intrinsic and center-of-mass contributions [107]. Equivalently, the intrinsic kinetic energy $T^{\prime}=T-T_{\mathrm{cm}}$ may thus be considered to have had the center-of-mass kinetic energy "subtracted out", as

$$
T^{\prime}=\frac{1}{2 m_{N}} \underbrace{\sum_{i} \mathbf{p}_{i} \cdot \mathbf{p}_{i}}_{\text {One-body }}-\frac{1}{2 A m_{N}} \underbrace{\left(\sum_{i} \mathbf{p}_{i}\right) \cdot\left(\sum_{j} \mathbf{p}_{j}\right)}_{\text {Center-of-mass }} .
$$

Then, while the lab-frame $T$ is a one-body operator, the intrinsic $T^{\prime}$ is a two-body operator. It is related, by an $A$-dependent counting factor, to the sum of two-nucleon relative kinetic energies, as

$$
T^{\prime}=\frac{1}{4 m_{N} A} \underbrace{\sum_{i j}^{\prime}\left(\mathbf{p}_{i}-\mathbf{p}_{j}\right) \cdot\left(\mathbf{p}_{i}-\mathbf{p}_{j}\right)}_{\text {Relative two-body }}
$$

where the primed sum omits diagonal $(i=j)$ terms. It may therefore be characterized as a relative two-body operator. The essential input for working with a two-body operator in a many-body calculation is its two-body matrix elements. The explicit relative two-body form (3) is well-suited for work with a harmonic oscillator basis. If the matrix elements are evaluated in an oscillator basis on the relative coordinate, then the full two-body matrix elements (between antisymmetrized products of harmonic oscillator single-particle wave functions) are readily obtained through the Moshinsky transformation [108, 109].

\footnotetext{
${ }^{1}$ Here, for simplicity, the same mass $m_{N}$ is taken for all nucleons, in practice commonly defined as $m_{N}=$ $\frac{1}{2}\left(m_{p}+m_{n}\right)$, but more properly taken as $m_{N}=\left(Z m_{p}+N m_{n}\right) / A$ [105, 106]. The full intrinsic kinetic energy operator, with explicit dependence on the nucleon masses, is treated in Appendix $B$.
} 
Finally, $T^{\prime}$ may be decomposed into one-body and two-body separable contributions as

$$
T^{\prime}=\frac{1}{2 m_{N}}\left(1-\frac{1}{A}\right) \underbrace{\sum_{i} \mathbf{p}_{i} \cdot \mathbf{p}_{i}}_{\text {One-body }}-\frac{1}{2 m_{N} A} \underbrace{\sum_{i j}^{\prime} \mathbf{p}_{i} \cdot \mathbf{p}_{j}}_{\text {Two-body separable }} .
$$

The one-body matrix elements of the one-body term are readily evaluated as radial integrals, while two-body matrix elements of a separable operator are readily evaluated as products of one-body matrix elements via Racah's reduction formula [110] (see discussions for the intrinsic kinetic energy, in particular, in Refs. [91, 93]). This approach makes no assumptions as to the form of the radial wave functions for the single-particle states, and thus its relevance is not confined to the harmonic oscillator basis.

To illustrate calculation of an intrinsic observable, let us take the root-mean-square (r.m.s.) radius of the nucleus. The expectation value $\left\langle\Psi\left|\left(\sum_{i} \mathbf{x}_{i} \cdot \mathbf{x}_{i}\right)\right| \Psi\right\rangle$, yields the summed squared radius of the nucleon probability distribution in the laboratory frame, that is, around the arbitrary coordinate origin introduced in defining the many-body problem. A laboratoryframe r.m.s. radius is then obtained as $r=\left[A^{-1}\left\langle\Psi\left|\left(\sum_{i} \mathbf{x}_{i} \cdot \mathbf{x}_{i}\right)\right| \Psi\right\rangle\right]^{1 / 2}$. Since the operator entering into the expectation value is a one-body operator, this expectation value is readily calculated in second-quantized formalism.

The nucleon density distribution in the laboratory frame, however, reflects both the intrinsic distribution of nucleons relative to the nuclear center of mass and the excursions of this center of mass relative to the origin. (Indeed, if the motion of the nucleons cleanly factorizes into intrinsic and center-of-mass parts, then the laboratory-frame density is simply the convolution of the nucleon density in the translational intrinsic frame and the probability density of the center-of-mass wave function with respect to the center-of-mass coordinate.)

The r.m.s. radius accessed in experiment is the intrinsic observable, arising only from the intrinsic structure relative to the center of mass. This intrinsic radius is instead obtained from an expectation value defined in terms of intrinsic coordinates as $r^{\prime}=$ $\left[A^{-1}\left\langle\Psi\left|\left(\sum_{i} \mathbf{x}_{i}^{\prime} \cdot \mathbf{x}_{i}^{\prime}\right)\right| \Psi\right\rangle\right]^{1 / 2}$. This expectation value now involves a two-body operator, of the same bilinear form as the intrinsic kinetic energy above. Relations directly analogous to (1)-(4) above apply to the intrinsic radius, along with the corresponding observations about evaluating two-body matrix elements. Although we have taken the density of the full nucleon distribution here, including both protons and neutrons, analogous arguments apply to calculating the root-mean-square radius of the proton distribution relative to the center of mass, and thus the nuclear charge radius (e.g., Refs. 92, 94]).

\section{B. Many-body space and symmetry structure}

In symmetry-adapted formulations of the nuclear many-body problem [111, based on the $\mathrm{U}(3)$ or $\mathrm{Sp}(3, \mathbb{R})$ groups, intrinsic operators have a fundamental relationship to the structure of the space in which many-body calculations are performed. The very definition of the symmetry-adapted many-body space and its truncation schemes depend upon the existence of intrinsic realizations of the group generators, and the calculational machinery may make use of the intrinsic group structure as well.

The group $\operatorname{Sp}(3, \mathbb{R})$ is generated by the full set of bilinear operators in coordinates and/or momenta. Elliott's U(3) subgroup, the harmonic oscillator degeneracy group, is generated by those linear combinations of bilinears which conserve the number of oscillator quanta. 
This $\mathrm{U}(3)$ group decomposes as $\mathrm{U}(3)=\mathrm{U}(1) \times \mathrm{SU}(3)$. Here $\mathrm{U}(1)$ is simply the trivial Abelian group of the harmonic oscillator Hamiltonian. The generators of $\mathrm{SU}(3)$ may be taken as the components $L_{1 M}$ of the orbital angular momentum and the components $\mathcal{Q}_{2 M}$ of a quadrupole tensor. The orbital angular momentum group $\mathrm{SO}(3)$ is thus a subgroup. The generators of $U(3)$ and $\operatorname{Sp}(3, \mathbb{R})$ are reviewed in Sec. A 4 .

In a symmetry-adapted approach, the basis for the nuclear many-body space is chosen to reflect the organization of this space into irreducible representations (irreps) either of the $\mathrm{U}(3)$ group chain $\mathrm{U}(3)=\mathrm{U}(1) \times[\mathrm{SU}(3) \supset \mathrm{SO}(3)]$ or the full $\mathrm{Sp}(3, \mathbb{R})$ group chain $\mathrm{Sp}(3, \mathbb{R}) \supset \mathrm{U}(3)=\mathrm{U}(1) \times[\mathrm{SU}(3) \supset \mathrm{SO}(3)]$. A $\mathrm{U}(3)$ irrep is labeled by the $\mathrm{U}(1)$ quantum number $N$ together with the $\mathrm{SU}(3)$ quantum numbers $(\lambda, \mu)$, which taken together form a $\mathrm{U}(3)$ label $\omega=N(\lambda, \mu)$. A $\mathrm{U}(3)$ irrep then decomposes into $\mathrm{SO}(3)$ irreps, labeled by the orbital angular momentum $L$.

The intrinsic generators for $\mathrm{U}(3)[112$ and $\mathrm{Sp}(3, \mathbb{R})[96]$ are obtained by the same prescription described above, i.e., replacement of coordinates and momenta by their intrinsic-frame expressions. What is important is that, due to their bilinear structure, the laboratory-frame generators $G$ decompose into the intrinsic operators $G^{\prime}$ and center-of-mass operators $G_{\mathrm{cm}}$, as

$$
G=G^{\prime}+G_{\mathrm{cm}}
$$

The two sets of operators $\left\{G^{\prime}\right\}$ and $\left\{G_{\mathrm{cm}}\right\}$ are mutually commuting, and the operators within each set themselves respectively obey the commutation relations to form the generators for intrinsic and center-of-mass realizations of the group, that is, of $U(3)$ or $\operatorname{Sp}(3, \mathbb{R})$.

The implications of this intrinsic group structure are already familiar for the $\mathrm{SO}(3)$ orbital angular momentum subgroup. Recall the classical result that the angular momentum about the center of mass and the angular momentum of the center of mass add to give the total angular momentum [113. In the quantum treatment, the existence of mutually commuting intrinsic and center-of-mass angular momentum groups, $\mathrm{SO}_{\text {intr }}(3)$ and $\mathrm{SO}_{\text {c.m. }}(3)$, combining to give the total angular momentum group $\mathrm{SO}(3)$ via the sum generators $\mathbf{L}=\mathbf{L}^{\prime}+\mathbf{L}_{\text {c.m. }}$. ensures that the intrinsic and center-of-mass angular momenta of the many-body system combine as $L^{\prime} \times L_{\mathrm{cm}} \rightarrow L$, according to the usual rules of angular momentum addition, to yield the total angular momentum of the system.

Similarly, the existence of an intrinsic U(1) group structure underlies the traditional use of an $N_{\text {max }}$-truncated harmonic oscillator basis in ab initio no-core configuration interaction (NCCI) calculations [100]. An $N_{\max }$-truncated oscillator basis is built from antisymmetrized products of harmonic oscillator single-particle states and includes all product states with up to $N_{\max }$ total harmonic oscillator excitations relative to the lowest Pauli-allowed filling of oscillator shells [114]. With such a choice of basis, the many-body space separates cleanly as the direct sum of a subspace which is free of center-of-mass exitations and a complementary spurious space. Within the center-of-mass free subspace, all the wave functions factorize into center-of-mass and intrinsic parts and share a well-defined motion in the center-of-mass coordinate, described by a harmonic oscillator $0 s$ ground state wave function. This zero-point motion in the center-of-mass coordinate combines with some, in general, much more complicated structure in the intrinsic coordinates, all while retaining fermionic antisymmetry. (As a special case, the $0 \hbar \omega$ shell model space, consisting of the complete set of many-body states defined in a single oscillator major shell, is well-known to have pure $0 s$ center-of-mass motion [115.)

The $N_{\max }$ truncation scheme works as it does as the result of the intrinsic and centerof-mass harmonic oscillator $\mathrm{U}(1)$ groups, $\mathrm{U}_{\text {intr }}(1)$ and $\mathrm{U}_{\mathrm{c} . \mathrm{m} .}(1)$, respectively, combining to 
give the sum generator, or total number operator, $N=N^{\prime}+N_{\text {c.m. }}$. (see Sec. F 3). Since the number operators $N^{\prime}, N_{\text {c.m. }}$, and $N$ are mutually commuting, they may be simultaneously diagonalized in the harmonic oscillator many-body basis, yielding (in principle) a new basis consisting of simultaneous eigenstates of $N^{\prime}, N_{\text {c.m. }}$, and $N=N^{\prime}+N_{\text {c.m. }}$. This choice of basis decomposes the many-body space as a direct sum of subspaces with definite $N^{\prime}$ and $N_{\text {c.m. }}$., and truncation by $N$, as in the $N_{\max }$ scheme, preserves such a structure.

It is clearest to illustrate this transformation graphically, taking the example of ${ }^{4} \mathrm{He}$ with $N_{\max }=4 \int^{2}$ Each panel of Fig. 1 1 represents the block structure of the matrix realization of a Galilean-invariant intrinsic Hamiltonian operator, $H=T^{\prime}+V$, as we restructure the basis, which is indicated along the top edge of the matrix.

The naturally constructed oscillator basis is obtained by distributing nucleons over orbitals, each of which may be characterized by its major oscillator shell $N_{i}$ (where, given indistinguishable particles, we may take $N_{1} \geq N_{2} \geq \cdots \geq N_{A}$ without loss of generality). This basis is indicated in Fig. 1(a). The basis states are eigenstates of $N=\sum N_{i}$, and are shown grouped by $N$ in Fig. 1(a). However, they are not eigenstates of $N^{\prime}$ or $N_{\text {c.m. }}$ separately, and there are thus no selection rules on a Galilean-invariant operator.

Transforming to a basis obtained by simultaneously diagonalizing $N^{\prime}$ and $N_{\text {c.m. }}$. yields the basis indicated in Fig. 1(c). These states are obtained by a nontrivial unitary transformation of the original basis states (i.e., taking linear combinations, not simply rearranging and relabeling these states). The basis states are still eigenstates of $N$, so the unitary transformation takes place separately within each subspace of definite $N$, and the basis states are again shown grouped by $N$ in Fig. 1(c). A Galilean-invariant operator acts as the identity operator on the center-of-mass degree of freedom and thus cannot connect states of different $N_{\text {c.m. }}$, i.e., $\left\langle N_{\text {c.m. }}{ }^{\prime}\left|H^{\prime}\right| N_{\text {c.m. }}\right\rangle=0$ for $N_{\text {c.m. }}{ }^{\prime} \neq N_{\text {c.m. }}$. This selection rule yields the block-sparse structure shown in Fig. 11(c).

Rearranging the basis to be grouped instead by $N_{\text {c.m. }}$, as in Fig. 1(d), turns the blocksparse structure into a block-diagonal structure. This rearrangement of the basis defines a decomposition of the $N_{\max }=4$ space as a direct sum of subspaces, each the product of an intrinsic subspace (successively more and more truncated) with a center-of-mass subspace (involving successively higher $N_{\text {c.m. }}$ ):

$$
\mathcal{H}^{\left(N_{\max }=4\right)}=\{0,2,4\}_{\text {intr }} \times\{0\}_{\text {c.m. }}+\{0,2\}_{\text {intr }} \times\{2\}_{\text {c.m. }}+\{0\}_{\text {intr }} \times\{4\}_{\text {c.m. }} .
$$

The Galilean invariance of the operator dictates that matrix elements between states with identical intrinsic structure are identical, regardless of the spectator center-of-mass wave function. Therefore, each successive block along the diagonal effectively represents a truncation, or submatrix, of the preceeding block $\left.\right|^{3}$ This structure is emphasized by the hatch lines, in Fig. 1(d), which indicate the intrinsic quantum numbers entering into each block of the matrix.

The desired center-of-mass free states (with center-of-mass $0 s$ wave functions) arise from diagonalization of the first block along the diagonal. The remaining blocks yield the spurious states, which also reflect a poorer description of the intrinsic structure, obtained in a more

\footnotetext{
${ }^{2}$ For simplicity, we restrict attention to the even parity space, and thus even values of $N$, and furthermore restrict attention to intrinsic excitations of even parity, and thus even $N^{\prime}$. A parity-conserving Galileaninvariant Hamiltonian will not connect subspaces of even and odd $N^{\prime}$.

${ }^{3}$ Details of the actual choice of basis states within each subspace, in particular, imposing angular momentum coupling between the intrinsic and center-of-mass motion, could obscure this simple relationship.
} 
(a)

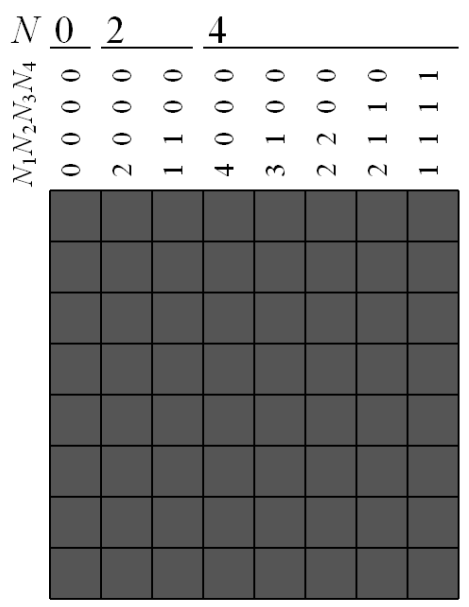

(c)

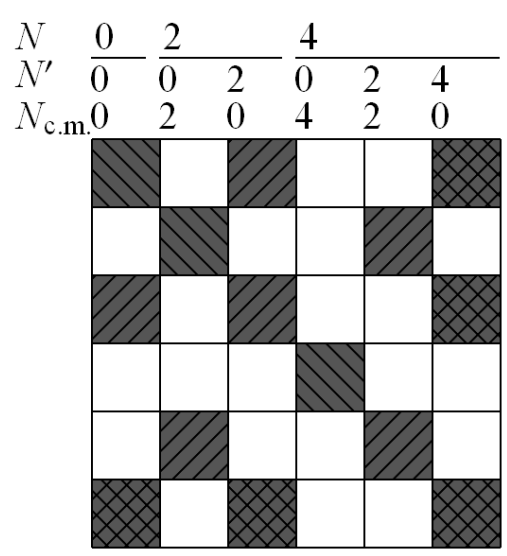

(b)

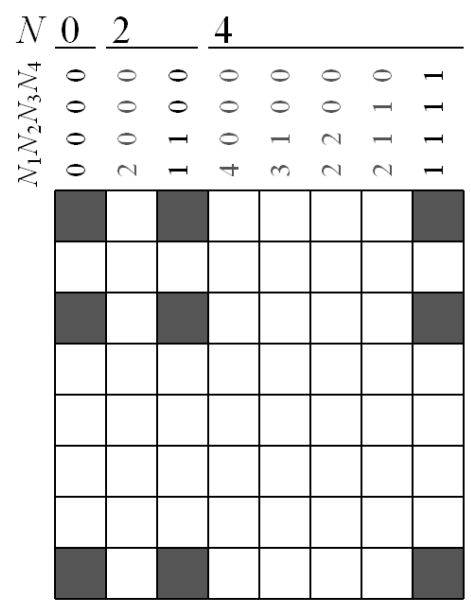

(d)

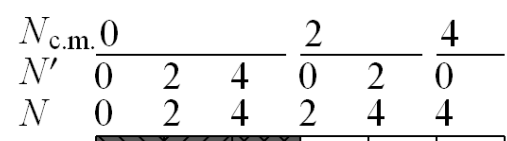

FIG. 1. Block structure of the matrix representation of a Galilean-invariant Hamiltonian, in the $N_{\text {max }}=4$ harmonic oscillator space of ${ }^{4} \mathrm{He}$ : (a) Represented in the natural oscillator-configuration basis, with occupied oscillator shells $N_{i}$, grouped by $N$. (b) Similarly, but restricted to the FCI subspace with $N_{i} \leq 1$. (c) Represented in a simultaneous eigenbasis of $N^{\prime}, N_{\text {c.m. }}$, and $N$, grouped by $N$. (d) Similarly, but grouped by $N_{\text {c.m. }}$. Block sizes are not proportional to actual subspace dimensions (which are 1 for $N=0,58$ for $N=2$, and 893 for $N=4$ in an $M=0 M$-scheme [116] basis for ${ }^{4} \mathrm{He}$ ). Hatching indicates the intrinsic structure entering into the matrix element, namely, whether the matrix element involves bra and ket states with $N^{\prime}=0$ (backward hatched), $N^{\prime} \leq 2$ (forward hatched), or $N^{\prime} \leq 4$ (cross hatched).

severely truncated subspace. For instance, considering the decomposition of the ${ }^{4} \mathrm{He} N_{\max }=$ 4 space in (6), the spectrum of $H^{\prime}$ in this space consists of three copies of the intrinsic excitation spectrum, each a truncated approximation to the untruncated intrinsic spectrum: the highest-fidelity rendition of the intrinsic spectrum, obtained in the $\{0,2,4\}_{\text {intr }}$ space and accompanied by no center-of-mass excitation; an intermediate-fidelity rendition of the intrinsic spectrum, obtained in the $\{0,2\}$ intr space and accompanied by $N_{\text {c.m. }}=2$ excitations; and a lowest-fidelity intrinsic spectrum, obtained in the $\{0\}_{\text {intr }}$ space and accompanied by $N_{\text {c.m. }}=4$ excitations.

It is to be emphasized that, in practical calculations, the basis states of definite $N^{\prime}$ and 
$N_{\text {c.m. }}$ [Fig. 1(c,d)] are never explicitly constructed. Rather, the mere fact that these basis states exist underlies the decomposition (6) of the truncated many-body space and thus the emergence of factorized eigenstates of definite $N_{\text {c.m. }}$. These factorized eigenstates simply come out of the diagonalization of the Galilean-invariant Hamiltonian.

In particular, to reap the benefits of the structure of the space, the Hamiltonian must be chosen so as to respect the block structure shown in Fig. 1(d). The original one-body kinetic energy $T$ connects oscillator basis states involving different center-of-mass excitations, i.e., $\left\langle N_{\text {c.m. }}{ }^{\prime}|T| N_{\text {c.m. }}\right\rangle \neq 0$, and in fact yields a block-tridiagonal structure in $N_{\text {c.m. }}$. It is this consideration that dictates its replacement by $T^{\prime}$, that is, use of an intrinsic Hamiltonian $H^{\prime}$, in NCCI calculations. The resulting low-lying spectrum then reflects the block structure described above, yielding multiple copies of the intrinsic spectrum, and these may be expected to appear at low energy, as the center-of-mass excitations do not carry any intrinsic kinetic energy. The spurious states carry no useful additional information about the intrinsic structure, serving only to pollute the calculated spectrum. However, the center-of-mass excited states may still be shifted out of the low-lying spectrum by addition of a center-of-mass Lawson term [116 118], proportional to $N_{\text {c.m. }}$, which preserves the block structure shown in Fig. 1(d). See Fig. 8 of Ref. [93] for an illustration both of the structure of the spurious spectrum and of the effect of the Lawson term.

In contrast, in calculations which depart from an $N_{\max }$-truncated oscillator many-body space, either by generalizing the oscillator truncation scheme [119] or starting from nonoscillator single-particle orbitals [93, 120], the decomposition (6) of the space in general may be expected to break down. This breakdown is illustrated for a full configuration-interaction (FCI) basis (e.g., Ref. [121]) in Fig. 11(b), where harmonic oscillator configurations are taken subject to a single-particle cutoff $N_{i} \leq 1$ on the occupied oscillator shells. The resulting space is a subspace of the $N_{\max }=4$ space. However, we no longer have a complete basis for each subspace of fixed $N$ (in particular, for the $N=2$ and 4 subspaces), which precludes the unitary transformation to the basis of Fig. 1(c).

Nonetheless, even without the exact factorization ensured by an $N_{\max }$-truncated oscillator basis, an approximate factorization of $s$-wave center-of-mass motion in the calculated eigenstates can still be obtained [93, 122, 123]. Even though the center-of-mass wave function is not directly accessible in an antisymmetrized products basis, to the extent that the center-of-mass wave function resembles $0 s$ harmonic oscillator zero-point motion, for some choice of the oscillator length parameter $b_{\mathrm{c} . \mathrm{m}}$. (Sec. F 3), this situation may be recognized by evaluating $\left\langle N_{\text {c.m. }}\right\rangle[93,122$, 123]. Approximate center-of-mass factorization may arise spontaneously, or it may be coerced by addition of a Lawson term, but doing so generally comes at the expense of convergence of the intrinsic wave function (fidelity to $0 s$ center-of-mass motion must be traded off against fidelity of the intrinsic wave function). See, e.g., Fig. 9 of Ref. [93] for an illustration of the resulting spectrum and use of the Lawson term for calculations in an antisymmetrized product basis of Laguerre functions [124-126].

With this understanding of the intrinsic oscillator structure of the many-body space in hand, let us now proceed to $\mathrm{U}(3)$. The intrinsic group $\mathrm{U}_{\text {intr }}(3)$ and center-of-mass group $\mathrm{U}_{\text {c.m. }}(3)$ are likewise mutually commuting. Consequently, irreps $\omega^{\prime}$ and $\omega_{\text {c.m. }}$, describing the intrinsic and center-of-mass motion, respectively, combine as $\omega^{\prime} \times \omega_{\text {c.m. }} \rightarrow \omega$ according to the usual $\mathrm{U}(3)$ coupling rules (e.g., Ref. [127]). These rules reduce to the addition of the $\mathrm{U}(1)$ quantum numbers $\left[N=N^{\prime}+N_{\text {c.m. }}\right]$ as above, and combination of the $(\lambda, \mu)$ quantum numbers according to the $\mathrm{SU}(3)$ coupling rules [128].

The existence of mutually commuting intrinsic and center-of-mass U(3) groups ensures 
(a) $N$

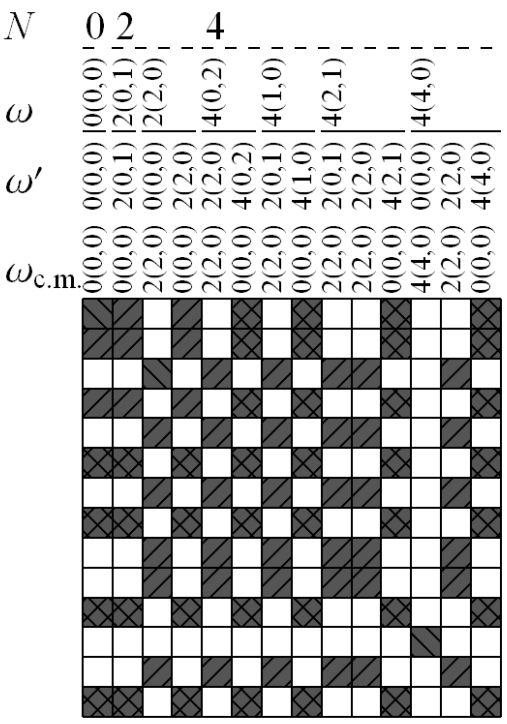

(b)

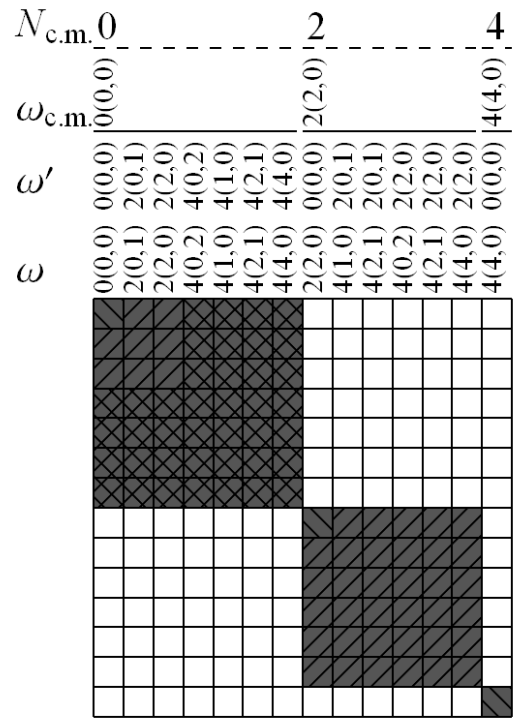

FIG. 2. Block structure of the matrix representation of Galilean-invariant Hamiltonian, in the $N_{\max }=4$ harmonic oscillator space of ${ }^{4} \mathrm{He}$, further decomposed into U(3) subspaces: (a) Represented in a basis of definite $\omega^{\prime}, \omega_{\text {c.m. }}$ (or, equivalently, $N_{\text {c.m. }}$ ), and $\omega$, grouped by $\omega$. (b) Similarly, but grouped by $\omega_{\text {c.m. }}$ (or, equivalently, $N_{\text {c.m. }}$ ). Hatching indicates whether the matrix element involves bra and ket states with $N^{\prime}=0$ (downward sloped hatching), $N^{\prime} \leq 2$ (upward sloped hatching), or $N^{\prime} \leq 4$ (cross hatching).

that, if the many-body space is truncated according to $\mathrm{U}(3)$ quantum numbers, a decomposition of the space into products of intrinsic and center-of-mass subspaces can again be carried out. The many-body space can be simultaneously decomposed into irreps of the intrinsic, center-of-mass, and total U(3) groups (this may be thought of as simultaneously diagonalizing the Casimir operators for the three groups), and a basis can (in principle) be obtained consisting of states of definite $\omega^{\prime}, \omega_{\text {c.m. }}$, and $\omega$. The decomposition in Fig. 11(c) is then broken down more finely by inclusion of additional quantum numbers, but the reorganization into block-diagonal form by $N_{\text {c.m. }}$, as in Fig. 11(d), can still be carried out. Thus, notably, the factorization of the resulting wave functions into intrinsic and center-of-mass factors, and the extraction of center-of-mass free states, can again be obtained, even if the space is truncated not just by total $N$ but more finely by total $\omega$ [112, 129133 .

In particular, the natural basis for a U(3)-coupled NCCI calculation, as in the symmetryadapted NCSM (SA-NCSM) of Refs. [134, 135], is obtained by distributing nucleons over oscillator shells, yielding definite total $N$, then ensuring that the resulting states are coupled to give good total $\omega$. The separability property guarantees that factorized center-of-mass motion will be obtained if an intrinsic Hamiltonian (with optional Lawson term) is diagonalized in any such basis selected to include all basis states of a given set of total $\mathrm{U}(3)$ quantum numbers $\omega$. Diagonalization in the traditional $N_{\max }$-truncated oscillator space is recovered as the special case in which all $\mathrm{U}(3)$ irreps up to a given $N$ are retained.

We illustrate the reorganization of the many-body space by intrinsic and total $\mathrm{U}(3)$ quantum numbers, again for the $N_{\max }=4$ space of ${ }^{4} \mathrm{He}$, in Fig. 2. For the intrinsic U(3), the possible irreps with $N^{\prime} \leq 4$ (even) have $\omega^{\prime}=0(0,0), 2(0,1), 2(2,0), 4(0,2), 4(1,0)$, $4(2,1)$, and $4(4,0)$, and, as it turns out, the same values are obtained for the $\omega$ of the 
total $\mathrm{U}(3) \mathrm{H}^{4}$ For the center-of-mass degree of freedom, the $\mathrm{U}(3)$ quantum numbers are trivially related to the number of oscillator quanta, as $\omega_{\text {c.m. }}=N_{\text {c.m. }}\left(N_{\text {c.m. }}, 0\right)$. The only nontrivial $\mathrm{U}(3)$ couplings entering into Fig. 2 are for the coupling of the $N^{\prime}=2$ intrinsic irreps to the $N_{\text {c.m. }}=2$ center-of-mass motion: $2(0,1) \times 2(2,0) \rightarrow 4(1,0), 4(2,1)$ and $2(2,0) \times$ $2(2,0) \rightarrow 4(0,2), 4(2,1), 4(4,0)$. The resulting basis states are grouped by $\omega$ in Fig. 2(a), giving block-sparse structure for a Galilean-invariant operator. These basis states are then rearranged by $N_{\text {c.m. }}$ (or, equivalently, $\omega_{\text {c.m. }}$ ) in Fig. 2(b), giving block-diagonal structure for a Galilean-invariant operator. Observe that truncating the $N_{\max }=4$ space by $\omega$, for instance, eliminating the $\omega=4(0,2)$ and $4(1,0) \mathrm{U}(3)$ subspaces, would reduce (subset) the set of $\omega$ values included in Fig. 2(a), and would thus eliminate certain rows and columns from within the block-diagonal structure of Fig. 2(b). However, it would not destroy this block-diagonal structure, nor would it interfere with the decomposition of the space into intrinsic and center-of-mass product subspaces that this structure implies.

In the SpNCCI framework [101-103], the nuclear many-body calculation is carried out in a center-of-mass free $\operatorname{Sp}(3, \mathbb{R})$ basis. Matrix elements of the intrinsic Hamiltonian (and other intrinsic operators for observables) in this basis are computed using a recurrence relation derived from the commutation relations between the intrinsic $\operatorname{Sp}(3, \mathbb{R})$ generators and certain $\mathrm{SU}(3)$-coupled unit tensor operators which provide a basis for the space of operators. The approach builds on ideas of Reske, Suzuki, and Hecht [136 138.

Seed matrix elements for the recurrence are obtained via the usual second-quantized approach, on U(3)-coupled SA-NCSM basis states with definite $0 s$ center-of-mass motion (these may be obtained either by diagonalizing the center-of-mass number operator $N_{\text {c.m. }}$.

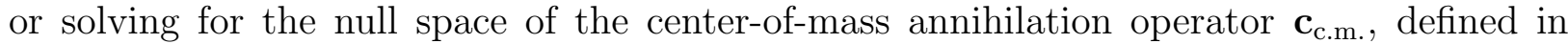
Sec. F 3). The recurrence process then bypasses the need for any further reference to the laboratory-frame single-particle representation of the many-body problem. Since the seed matrix elements involve center-of-mass free states, and since the recurrence is obtained by implicitly acting on these states with intrinsic $\operatorname{Sp}(3, \mathbb{R})$ raising generators $A^{\prime(2,0)}$, the resulting matrix elements are those on a center-of-mass free $\operatorname{Sp}(3, \mathbb{R})$ basis, although this basis need never be explicitly constructed in terms of laboratory-frame states. The calculations rely upon the relations, developed in the present work, between the defining expressions for the intrinsic bilinear operators and their explicit two-body forms [101].

Regardless of how "center-of-mass free" solutions to the many-body problem are obtained, whether as a byproduct of diagonalizing a Galilean-invariant Hamiltonian or a priori through construction of a center-of-mass free basis for the problem, it is important to note that imposing a well-defined $0 s$ zero-point center-of-mass motion is not to be conflated with complete removal of the center-of-mass degree of freedom from the problem. Unless care is taken to work in terms of intrinsic operators for observables, calculated observables may in general be expected to reflect contamination from this zero-point center-of-mass motion. A case in point is provided by the r.m.s. radius, as discussed in Sec. II A. The squared radius evaluated with the laboratory-frame operator, in a center-of-mass free state, measures a density which is the convolution of the density in the intrinsic frame with the Gaussian profile of the zero-point motion of the center of mass [139]. In this case, the required correction is straightforward [see $(\mathrm{A} 7 \mathrm{D})]$.

While there are certain restricted circumstances, involving center-of-mass free wave functions, under which the naive one-body laboratory-frame transition operators can be used

\footnotetext{
${ }^{4}$ These values may be read off from Fig. 5(a) of Ref. [133].
} 
(as they indeed often are) without introducing center-of-mass contamination to calculated observables, even here, an understanding these circumstances requires an understanding of the intrinsic forms of these operators (see Secs. IV] and V).

\section{DEFINITIONS: OPERATORS AND COORDINATES}

\section{A. One-body and two-body operators}

One-body operators are operators on the $A$-particle space, but they are uniquely defined by their action on the single-particle space. Similarly, two-body operators are operators on the $A$-particle space, but they are uniquely defined by their action on the two-particle space. For fermionic problems, in particular, the matrix elements of one-body or two-body operators in a basis of antisymmetrized product states (Slater determinants) on the $A$-particle space can readily be computed from the matrix elements of these operators between basis states for the one-particle or two-particle spaces, respectively, through second quantization (see, e.g., Ref. [140]). Thus, in setting up a many-body calculation involving these operators, it is only necessary to evaluate the relevant matrix elements on the one-particle or two-particle spaces, respectively. These one-body and two-body matrix elements then serve as the input to the standard computational machinery of the many-body calculation, which can generate the matrix elements of these operators in the $A$-particle antisymmetrized product basis.

As our focus in this work is to establish relations between different one-body and twobody decompositions of intrinsic operators, we first, in this section, establish definitions for these operators. In particular, we must have an unambiguous notation for relating operators on the one-particle and two-particle spaces with the corresponding one-body and two-body operators on the $A$-particle space, respectively. A one-body operator, acting on the $A$-particle space, is obtained by taking an operator $u$ on the single-particle space and applying it uniformly to all $A$ particles:

$$
U[u]=\sum_{i} u_{i}
$$

where $u_{i}$ acts as $u$ on the single-particle space of the $i$ th particle and as the identity for all other particles.

Similarly, a two-body operator, acting on the $A$-particle space, is obtained by taking an operator $v$ on the two-particle space and applying it uniformly to all pairs of $A$ particles:

$$
V[v]=\frac{1}{2} \sum_{i j}^{\prime} v_{i j},
$$

where $v_{i j}$ acts as $v$ on the two-particle space of the $i$ th and $j$ th particles and as the identity for all other particles. Here the prime on the sum indicates omission of diagonal terms (i.e., summation over $i, j=1, \ldots, A$ subject to the restriction $i \neq j)$. In this definition, the condition is imposed that the operator $v$ must be symmetric under interchange of particles in the two-particle space $\left(v_{12}=v_{21}\right)$, to ensure that $V[v]$ is symmetric under interchange of particle indices in the $A$-particle space.

While the distinction between one-body and two-body operators is clear when the operators are acting on the full Fock space, with arbitrary number of particles, the distinction is not as clear on a space of fixed particle number $A$. In fact, any one-body operator may be 
"upgraded" to an equivalent two-body operator via the summation identities in Appendix C. In particular, the one body operator $U[u]$ may be reexpressed [see $[\mathrm{C} 2]$ a $\mathrm{s}^{5}$

$$
U[u]=\frac{1}{(A-1)} V\left[u_{1}+u_{2}\right],
$$

on the $A$-particle space, where $u_{1}+u_{2}\left(=v_{12}\right)$ is the operator on the two-particle space obtained by the one-body action of $u$ on this space. (However, the converse does not hold, in that a two-body operator cannot, in general, be reduced to an equivalent one-body operator.) Such interconversion between one-body and two-body forms of an operator are essential to the relations obtained in this work.

\section{B. Relative coordinates (two-body system)}

Relative and c.m. coordinates for the two-body system are proportional to the difference $\mathbf{x}_{1}-\mathbf{x}_{2}$ and sum $\mathbf{x}_{1}+\mathbf{x}_{2}$ of single-particle coordinates, respectively, and similarly for the momenta. However, there is freedom in the choice of conventional factors in these definitions, arising from the freedom to carry out a canonical transformation on the coordinates and momenta. We therefore review the definitions so that we may avoid ambiguity in the transformation between a relative operator on the two-particle space (which will be expressed in terms of the relative coordinate $\mathbf{x}_{\text {rel }}$ and momentum $\mathbf{k}_{\text {rel }}$ ) and the corresponding intrinsic operator on the $A$-particle space obtained via (8), as in the example (13).

In traditional mechanics applications, it is natural to take the relative coordinate $\mathbf{x}_{\text {rel }}$ as the displacement between particles and the c.m. coordinate $\mathbf{x}_{\mathrm{c} . \mathrm{m}}$. as the mean of the coordinate vectors, that is, quite literally the "center of the masses" (at least in the case of equal masses, which we consider here):

$$
\begin{array}{ll}
\mathbf{x}_{\text {rel }}=\left(\mathbf{x}_{1}-\mathbf{x}_{2}\right) & \mathbf{x}_{\text {c.m. }}=\frac{1}{2}\left(\mathbf{x}_{1}+\mathbf{x}_{2}\right) \\
\mathbf{k}_{\text {rel }}=\frac{1}{2}\left(\mathbf{k}_{1}-\mathbf{k}_{2}\right) & \mathbf{k}_{\text {c.m. }}=\left(\mathbf{k}_{1}+\mathbf{k}_{2}\right) .
\end{array}
$$

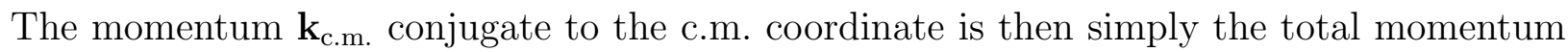
of the system (the normalizations for the conjugate momenta, $\mathbf{k}_{\mathrm{rel}}$ and $\mathbf{k}_{\mathrm{c} . \mathrm{m} \text {. }}$, are forced from the definitions of $\mathbf{x}_{\text {rel }}$ and $\mathbf{x}_{\text {c.m. }}$, by the requirement of conjugacy).

However, in working with the quantum harmonic oscillator, it is natural to recognize the duality between coordinates and momenta more explicitly, by symmetrically distributing the coefficients of $1 / 2$ between the coordinate and the momentum, as adopted in, e.g., Ref. [109]. This also provides greater parallelism between the treatment of the relative and c.m. degrees of freedom:

$$
\begin{array}{ll}
\mathbf{x}_{\text {rel }}=\frac{1}{\sqrt{2}}\left(\mathbf{x}_{1}-\mathbf{x}_{2}\right) & \mathbf{x}_{\text {c.m. }}=\frac{1}{\sqrt{2}}\left(\mathbf{x}_{1}+\mathbf{x}_{2}\right) \\
\mathbf{k}_{\text {rel }}=\frac{1}{\sqrt{2}}\left(\mathbf{k}_{1}-\mathbf{k}_{2}\right) & \mathbf{k}_{\text {c.m. }}=\frac{1}{\sqrt{2}}\left(\mathbf{k}_{1}+\mathbf{k}_{2}\right) .
\end{array}
$$

For results which are dependent upon the choice of convention (10) or (11) for the relativec.m. coordinates and momenta, we shall indicate the appropriate coefficients obtained under

\footnotetext{
${ }^{5}$ This relation (9) between $U[u]$ and $V\left[u_{1}+u_{2}\right]$ is an $A$-dependent relation, valid separately on each $A$ particle space, not a true $A$-independent operator identification. Thus, e.g., if $U[u]$ is to be treated as a two-body operator in a many-body computation, the two-body matrix elements evaluated for the $A=2$ basis must then be rescaled by $1 /(A-1)$ to be applied on the $A$-particle problem.
} 
each of the two conventions in braces, with the upper value for mechanics convention and the lower value for the symmetric convention. Thus, e.g., the definitions (10) or (11) may be expressed together as

$$
\begin{array}{ll}
\mathbf{x}_{\mathrm{rel}}=\left\{\begin{array}{c}
1 \\
\frac{1}{\sqrt{2}}
\end{array}\right\}\left(\mathbf{x}_{1}-\mathbf{x}_{2}\right) & \mathbf{x}_{\mathrm{c} . \mathrm{m} .}=\left\{\begin{array}{c}
\frac{1}{2} \\
\frac{1}{\sqrt{2}}
\end{array}\right\}\left(\mathbf{x}_{1}+\mathbf{x}_{2}\right) \\
\mathbf{k}_{\mathrm{rel}}=\left\{\begin{array}{c}
\frac{1}{2} \\
\frac{1}{\sqrt{2}}
\end{array}\right\}\left(\mathbf{k}_{1}-\mathbf{k}_{2}\right) & \mathbf{k}_{\mathrm{c} . \mathrm{m} .}=\left\{\begin{array}{c}
1 \\
\frac{1}{\sqrt{2}}
\end{array}\right\}\left(\mathbf{k}_{1}+\mathbf{k}_{2}\right) .
\end{array}
$$

Given that we will be focusing on Galilean-invariant systems and intrinsic observables, we will have special interest in relative two-body operators. These are two-body operators defined in terms of an operator $v$ on the two-particle space which is itself Galilean-invariant and, thus, if represented in terms of relative and center-of-mass (c.m.) coordinates (Sec. III B), can only involve the relative coordinate, not the c.m. coordinate. As a concrete illustration of the notation (8) for two-body operators, consider the squared relative momentum operator on the two-body system, which is, to within the conventional factors defined above in (12), given by $\mathbf{k}_{\mathrm{rel}}^{2} \propto\left(\mathbf{k}_{1}-\mathbf{k}_{2}\right)^{2}$. Then the corresponding two-body operator obtained on the $A$-particle space is

$$
V\left[\mathbf{k}_{\mathrm{rel}}^{2}\right] \propto V\left[\left(\mathbf{k}_{1}-\mathbf{k}_{2}\right)^{2}\right]=\frac{1}{2} \sum_{i j}^{\prime}\left(\mathbf{k}_{i}-\mathbf{k}_{j}\right)^{2} .
$$

\section{Intrinsic coordinates (many-body system)}

We now turn to coordinates for the $A$-particle system. These are, naturally, denoted by $\mathbf{x}_{i}(i=1, \ldots, A)$, with conjugate momenta $\mathbf{p}_{i}(i=1, \ldots, A)$ or, as we shall equivalently use in their place in the following discussion, wave vectors $\mathbf{k}_{i}(i=1, \ldots, A)$, where $\mathbf{p}_{i}=\hbar \mathbf{k}_{i}$. However, to define an observable in terms of coordinates and momenta, in such a way that it is manifestly Galilean-invariant quantity, we work instead with intrinsic coordinates $\mathbf{x}_{i}^{\prime}$ and intrinsic momenta $\mathbf{k}_{i}^{\prime}$ defined with respect to the system's center-of-mass frame.

The defining property of these intrinsic coordinates and momenta is that $M^{-1} \sum_{i} m_{i} \mathbf{x}_{i}^{\prime}=$ 0 (i.e., the center of mass is at the origin) and $\sum_{i} \mathbf{k}_{i}^{\prime}=0$ (i.e., the total momentum of the system vanishes), where $m_{i}$ are the masses, and $M=\sum_{i} m_{i}$. The transformation to intrinsic coordinates and momenta is obtained, for general choices of the masses $m_{i}$, as [113]

$$
\begin{array}{ll}
\mathbf{x}_{i}^{\prime}=\mathbf{x}_{i}-\quad \mathbf{x}_{\text {c.m. }} . & \mathbf{x}_{\text {c.m. }}=\frac{1}{M} \sum_{i} m_{i} \mathbf{x}_{i} \\
\mathbf{k}_{i}^{\prime}=\mathbf{k}_{i}-\frac{m_{i}}{M} \mathbf{k}_{\text {c.m. } .} & \mathbf{k}_{\text {c.m. }}=\sum_{i} \mathbf{k}_{i} .
\end{array}
$$

However, for simplicity, we specialize to particles of equal mass, namely, taking $m_{i} \rightarrow m_{N}$ and $M \rightarrow A m_{N}$ (see footnote 1). We then have,

$$
\begin{array}{ll}
\mathbf{x}_{i}^{\prime}=\mathbf{x}_{i}-\quad \mathbf{x}_{\mathrm{c} . \mathrm{m} .} & \mathbf{x}_{\mathrm{c} . \mathrm{m} .}=\frac{1}{A} \sum_{i} \mathbf{x}_{i} \\
\mathbf{k}_{i}^{\prime}=\mathbf{k}_{i}-\frac{1}{A} \mathbf{k}_{\mathrm{c} . \mathrm{m} .} & \mathbf{k}_{\mathrm{c} . \mathrm{m} .}=\sum_{i} \mathbf{k}_{i} .
\end{array}
$$


Note that $\mathbf{x}_{\text {c.m. }}$. and $\mathbf{k}_{\text {c.m. }}$. may alternatively be defined, by canonical transformation, such that the factor of $1 / A$ is symmetrically distributed between them (see also Sec. [III B), giving

$$
\begin{array}{ll}
\mathbf{x}_{i}^{\prime}=\mathbf{x}_{i}-\frac{1}{\sqrt{A}} \mathbf{x}_{\text {c.m. }} & \mathbf{x}_{\text {c.m. }}=\frac{1}{\sqrt{A}} \sum_{i} \mathbf{x}_{i} \\
\mathbf{k}_{i}^{\prime}=\mathbf{k}_{i}-\frac{1}{\sqrt{A}} \mathbf{k}_{\text {c.m. } .} & \mathbf{k}_{\text {c.m. }}=\frac{1}{\sqrt{A}} \sum_{i} \mathbf{k}_{i} .
\end{array}
$$

For results which are dependent upon the choice of convention 15 or (16) for the c.m. coordinates and momenta, we shall indicate the appropriate coefficients obtained under each of the two conventions in braces, with the upper value for mechanics convention and the lower value for the symmetric convention. Thus, e.g., the definitions (15) or (16) themselves may be expressed together as

$$
\begin{array}{ll}
\mathbf{x}_{i}^{\prime}=\mathbf{x}_{i}-\left\{\begin{array}{c}
1 \\
1 / \sqrt{A}
\end{array}\right\} \mathbf{x}_{\mathrm{c} . \mathrm{m} .} & \mathbf{x}_{\mathrm{c} . \mathrm{m} .}=\left\{\begin{array}{c}
1 / A \\
1 / \sqrt{A}
\end{array}\right\} \sum_{i} \mathbf{x}_{i} \\
\mathbf{k}_{i}^{\prime}=\mathbf{k}_{i}-\left\{\begin{array}{c}
1 / A \\
1 / \sqrt{A}
\end{array}\right\} \mathbf{k}_{\mathrm{c.m} .} & \mathbf{k}_{\mathrm{c} . \mathrm{m} .}=\left\{\begin{array}{c}
1 \\
1 / \sqrt{A}
\end{array}\right\} \sum_{i} \mathbf{k}_{i} .
\end{array}
$$

However, either way, eliminating the intermediate reference to the c.m. coordinate and momentum gives simply

$$
\begin{aligned}
\mathbf{x}_{i}^{\prime} & =\mathbf{x}_{i}-\frac{1}{A} \sum_{j} \mathbf{x}_{j} \\
\mathbf{k}_{i}^{\prime} & =\mathbf{k}_{i}-\frac{1}{A} \sum_{j} \mathbf{k}_{j}
\end{aligned}
$$

for the intrinsic coordinates and momenta.

Note that the intrinsic coordinates $\mathbf{x}_{i}^{\prime}$ and momenta $\mathbf{k}_{i}^{\prime}$ do not satisfy canonical commutation relations (see Sec. F 3). Also, although the intrinsic coordinates $\mathbf{x}_{1}^{\prime}$ or $\mathbf{x}_{2}^{\prime}$ on the twoparticle space are simply related to the relative coordinate $\mathbf{x}_{\mathrm{rel}}$, one should not mistakenly presume that intrinsic coordinates simply reduce to relative coordinates on the two-particle space.

\section{DIPOLE OPERATOR}

It is instructive to first consider the dipole or $E 1$ operator [88], as a simpler case, before moving on to the bilinear operators which serve as the main focus of the present work. The dipole operator allows us to illustrate several ingredients which arise in the treatment of bilinear operators, but without the distraction of some more cumbersome algebra. In particular, the following discussion of the dipole operator provides examples of transforming an operator to intrinsic coordinates, decomposing it into isoscalar and isovector contributions, decomposing an intrinsic operator into the "naive" one-body operator and a c.m. "recoil" contribution, and expressing an intrinsic operator as a pure relative two-body operator.

The mass, or isoscalar, dipole operator, which we define as the one-body operator

$$
\mathbf{D}=U[\mathbf{x}]=\sum_{i} \mathbf{x}_{i},
$$


is simply proportional to the c.m. coordinate $\mathbf{x}_{\text {c.m. }}$ (17) of the $A$-body system. It therefore comes as no surprise that, in the intrinsic frame, this operator vanishes identically. That is, if we attempt to construct an intrinsic dipole operator $\mathbf{D}^{\prime}$ by the substitution $\mathbf{x}_{i} \rightarrow \mathbf{x}_{i}^{\prime}(17)$, we obtain

$$
\begin{aligned}
\mathbf{D}^{\prime} & =\sum_{i}\left(\mathbf{x}_{i}-\frac{1}{A} \sum_{j} \mathbf{x}_{j}\right) \\
& =\sum_{i} \mathbf{x}_{i}-\frac{1}{A}\left(\sum_{i} 1\right)\left(\sum_{j} \mathbf{x}_{j}\right) \\
& =0
\end{aligned}
$$

where we have recognized that summing over a "free" particle index which does not appear in the summand simply introduces a counting factor $A\left(\sum_{i} 1=A\right)$.

Let us therefore move on to the variants of the dipole operator which distinguish protons and neutrons. We may define a proton dipole operator $\mathbf{D}_{p}$, in which the summation over particles runs only over protons, as $\mathbf{D}_{p}=\sum_{i \in p} \mathbf{x}_{i}$ (this proton dipole operator is, to within multiplication by the electric charge $e$, the physical electric dipole operator). We may similarly define a neutron dipole operator $\mathbf{D}_{n}$, in which the summation runs only over neutrons, as $\mathbf{D}_{n}=\sum_{i \in n} \mathbf{x}_{i}$. However, by invoking a restricted sum over particles, we have violated the defining property (7) of a one-body operator, as one which takes the action of an operator defined on the single-particle space, and sums this action over all $A$ particles. We thus instead allow the summation to range over all $A$ particles, but modify the definition of the operator on the single-particle space, so that it "sees" only protons, and vanishes when acting on neutrons, or vice versa. That is, we have

$$
\begin{aligned}
& \mathbf{D}_{p}=U\left[\mathbf{x} \delta_{p}\right]=\sum_{i} \mathbf{x}_{i} \delta_{i, p} \\
& \mathbf{D}_{n}=U\left[\mathbf{x} \delta_{n}\right]=\sum_{i} \mathbf{x}_{i} \delta_{i, n},
\end{aligned}
$$

where $\delta_{p}$ is an operator defined on the single-particle space, which acts as the identity on a proton and vanishes acting on a neutron, and vice versa for $\delta_{n}$. Therefore, in the sum, $\delta_{i, p}=1$ if the $i$ th particle is a proton or 0 if the particle is a neutron, and vice versa for $\delta_{i, n}$.

The isospin formalism provides the natural framework for defining such proton and neutron selection operators, and then allows us to decompose the proton and neutron dipole operators into isoscalar and isovector contributions. Following common practice in nuclear theory, we work with the Pauli matrix operators $\boldsymbol{\tau}$ for the nucleons; these are twice the isospin operators $(\boldsymbol{\tau}=2 \mathbf{t})$. We adopt the convention that $t_{3}=+1 / 2$ for the proton and $t_{3}=-1 / 2$ for the neutron, so $\tau_{0}\left(=\tau_{3}\right)$ has eigenvalues +1 for the proton and -1 for the neutron. Then $\delta_{p}=\frac{1}{2}\left(1+\tau_{0}\right)$ and $\delta_{n}=\frac{1}{2}\left(1-\tau_{0}\right)$. The expressions for proton and neutron dipole operators in (21) consolidate to

$$
\mathbf{D}_{\alpha}=U\left[\mathbf{x} \delta_{\alpha}\right]=\sum_{i} \mathbf{x}_{i} \delta_{i, \alpha}
$$

where we combine these definitions as

$$
\delta_{\alpha}=\frac{1}{2}\left(1+\alpha \tau_{0}\right)
$$

and use $\alpha=+1$ to select protons or $\alpha=-1$ to select neutrons. 
This proton or neutron dipole operator separates into manifestly isoscalar and isovector parts, as

$$
\mathbf{D}_{\alpha}=\frac{1}{2} \underbrace{\left[\sum_{i} \mathbf{x}_{i}\right]}_{\mathbf{D}}+\frac{1}{2} \alpha \underbrace{\left[\sum_{i} \mathbf{x}_{i} \tau_{i 0}\right]}_{\mathbf{D}_{\mathrm{IV}}} .
$$

That is, $\mathbf{D}_{\alpha}=\frac{1}{2} \mathbf{D}+\frac{1}{2} \alpha \mathbf{D}_{\mathrm{IV}}$, where $\mathbf{D}$ is simply the isoscalar dipole operator from (19), while

$$
\mathbf{D}_{\mathrm{IV}}=U\left[\mathbf{x} \tau_{0}\right]=\sum_{i} \mathbf{x}_{i} \tau_{i 0}
$$

is the isovector dipole operator. Since each term is manifestly the $T_{z}=0$ spherical tensor component of an isovector operator, this operator is itself, more precisely, the $T_{z}=0$ component of an isovector operator.

We focus now on the intrinsic formulation $\mathbf{D}_{\mathrm{IV}}^{\prime}$ of the isovector dipole operator. We have already found, in (20), that the intrinsic isoscalar dipole operator vanishes, so (24) implies that the intrinsic proton or neutron dipole operators $\mathbf{D}_{\alpha}^{\prime}$ are simply proportional to $\mathbf{D}_{\mathrm{IV}}^{\prime}$ :

$$
\mathbf{D}_{\alpha}^{\prime}=\frac{1}{2} \alpha \mathbf{D}_{\mathrm{IV}}^{\prime}
$$

Again substituting $\mathbf{x}_{i} \rightarrow \mathbf{x}_{i}^{\prime}$ (17) to obtain the intrinsic operator, we have

$$
\mathbf{D}_{\mathrm{IV}}^{\prime}=\sum_{i}\left(\mathbf{x}_{i}-\frac{1}{A} \sum_{j} \mathbf{x}_{j}\right) \tau_{i 0} .
$$

Observe that this expression for the intrinsic isovector dipole operator involves double sums over particle indices. The intrinsic operator is no longer purely a one-body operator but rather also includes two-body contributions.

Multiplying out the product of sums in (27) leaves us with an expression involving a double sum, as

$$
\mathbf{D}_{\mathrm{IV}}^{\prime}=\sum_{i} \mathbf{x}_{i} \tau_{i 0}-\frac{1}{A} \sum_{i j} \mathbf{x}_{j} \tau_{i 0} .
$$

The first term we recognize as simply the original, uncorrected one-body operator $\mathbf{D}_{\text {IV }}$ from (25), while the coordinate dependence of the remaining term involves only the centerof-mass degree of freedom:

$$
\underbrace{\mathbf{D}_{\mathrm{IV}}^{\prime}}_{\text {Intrinsic }}=\underbrace{\sum_{i} \mathbf{x}_{i} \tau_{i 0}}_{\text {One-body }}-\underbrace{\frac{1}{A}\left(\sum_{i} \tau_{i 0}\right)\left(\sum_{j} \mathbf{x}_{j}\right)}_{\text {Center-of-mass }} .
$$

Note that the isospin sum in the second term reduces to a simple counting factor $\left(\sum_{i} \tau_{i 0}=\right.$ $Z-N$, where $Z$ is the proton number, and $N$ is the neutron number), and we recognize that $\sum_{j} \mathbf{x}_{j}$ is simply $\mathbf{D} \propto \mathbf{x}_{\mathrm{c} . \mathrm{m} \text {. from } 19}$, giving

$$
\mathbf{D}_{\mathrm{IV}}^{\prime}=\mathbf{D}_{\mathrm{IV}}-\frac{Z-N}{A} \mathbf{D}
$$

Thus, the intrinsic operator so happens, in this particular case, to decompose into two separate terms: one representing the original "naive" one-body operator, and the other a 
c.m. (or "recoil") contribution. We highlight this separation (30), since a similar separation occurs below for the bilinear operators (Sec. V). While for the dipole operator considered here in (30) both terms are still simply one-body operators, for the bilinear operators considered below the c.m. contribution is a two-body operator.

As noted in Sec. II, for NCCI calculations in the traditional $N_{\max }$-truncated oscillator basis, the c.m. motion in the calculated many-body wave function is known to exactly factorize from the intrinsic motion, and the c.m. motion of the nuclear many-body state takes on a pure oscillator $0 s$ wave function. Since the $\mathbf{D}$ operator acts only on the c.m. degree of freedom, its matrix elements are then those of a vector operator between states of zero angular momentum, which vanish identically $[\langle 0 s\|\mathbf{D}\| 0 s\rangle=0]$ by the angular momentum selection rule. The matrix elements of the remaining terms $\mathbf{D}_{\mathrm{IV}}$ and $\mathbf{D}_{\mathrm{IV}}^{\prime}$ in 30 ) must therefore be equal. The analogous relation for the bilinear operators considered below plays a central role in practical calculations of matrix elements of, e.g., the $M 1$ and $E 2$ operators, between NCCI many-body eigenstates.

However, in more general varieties of many-body calculation, it cannot be assumed that the c.m. wave function separates or has such a simple form. We must therefore evaluate transition matrix elements of the full, two-body intrinsic isovector dipole operator between many-body wave functions.

Before we can evaluate two-body matrix elements, it is first necessary to represent the operator in the canonical form $V[v]$ for a two-body operator, defined in (8), so that the operator $v$ on the two-body space can be identified. We thus return to the expression for $\mathbf{D}_{\mathrm{IV}}^{\prime}$ in (28) and recast it manifestly in the form of (8). We must eliminate the one-body term (single sum), but first eliminate the diagonal $(i=j)$ terms from the double sum, and furthermore ensure that the summand in the double sum is symmetric in the particle indices, as required of $v_{i j}$. These tasks are accomplished by applying the summation identities from Appendix C, successively (C4) and (C1), yielding

$$
\mathbf{D}_{\text {IV }}^{\prime}=\frac{1}{2 A} \sum_{i j}^{\prime}\left(\mathbf{x}_{i} \tau_{i 0}+\mathbf{x}_{j} \tau_{j 0}\right)-\frac{1}{2 A} \sum_{i j}^{\prime}\left(\mathbf{x}_{i} \tau_{j 0}+\mathbf{x}_{j} \tau_{i 0}\right) .
$$

Within each of these two sums, the summand is manifestly symmetric under interchange of particle indices $i \leftrightarrow j$, so we have indeed obtained a two-body operator in (31).

Moreover, $\mathbf{D}_{\mathrm{IV}}^{\prime}$ is constructed as an intrinsic operator, and thus Galilean-invariant, so recall (Sec. III A) that we expect it to, more specifically, be a relative two-body operator, and the corresponding operator $v_{12}$ on the two-particle space should have a coordinate dependence which involves only the relative coordinate degrees of freedom. This is not obvious from (31), but refactoring yields

$$
\mathbf{D}_{\mathrm{IV}}^{\prime}=\frac{1}{2 A} \sum_{i j}^{\prime}\left(\mathbf{x}_{i}-\mathbf{x}_{j}\right)\left(\tau_{i 0}-\tau_{j 0}\right),
$$

with the appropriate coordinate dependence of the form $\mathbf{x}_{i}-\mathbf{x}_{j}$.

To explicitly recognize $\mathbf{D}_{\mathrm{IV}}^{\prime}$ as a two-body operator, following (8), we write

$$
\mathbf{D}_{\mathrm{IV}}^{\prime}=\frac{2}{A} V\left[\frac{1}{2}\left(\mathbf{x}_{1}-\mathbf{x}_{2}\right)\left(\tau_{10}-\tau_{20}\right)\right] .
$$

It is natural to pull all $A$ dependence outside of the definition of the two-body operator $V[\cdots]$ appearing in (33), so that this two-body operator can be defined independent of the number 
of nucleons $A$ in the many-body problem, and, for practical many-body calculations, its twobody matrix elements can therefore be evaluated independent of the number of particles $A$ targeted in the many-body calculation. Rather, we need only evaluate the matrix elements of the relative operator

$$
\mathbf{D}_{\text {IV }, \text { rel }}=\frac{1}{2}\left(\mathbf{x}_{1}-\mathbf{x}_{2}\right)\left(\tau_{10}-\tau_{20}\right),
$$

defined on the two-particle space. Matrix elements of the corresponding two-body operator $V\left[\mathbf{D}_{\mathrm{IV}, \text {,rel }}\right]$ on the many-particle space then follow as usual by the machinery of second quantization. The two-body matrix elements of the relative operator $\mathbf{D}_{\mathrm{IV} \text {,rel }}$ must then simply be scaled by the "counting factor" $2 / A$ to give two-body matrix elements for the intrinsic operator $\mathbf{D}_{\mathrm{IV}}^{\prime}$.

Note that we can explicitly represent this relative operator $\mathbf{D}_{\mathrm{IV} \text {,rel }}$ in terms of the relative coordinate $\mathbf{x}_{\mathrm{rel}}$, but we must take care that the expression depends on the choice of convention in the definition (12) of $\mathbf{x}_{\text {rel }}$ :

$$
\mathbf{D}_{\text {IV,rel }}=\left\{\frac{1}{\sqrt{2}}\right\} \frac{1}{2} \mathbf{x}_{\text {rel }}\left(\tau_{10}-\tau_{20}\right) .
$$

Also, note that the isospin dependence $\tau_{10}-\tau_{20}$ enters as the zero component of the isovector operator $\boldsymbol{\tau}_{1}-\boldsymbol{\tau}_{2}$, justifying the identification of $\mathbf{D}_{\mathrm{IV}, \text { rel }}$, and hence $\mathbf{D}_{\mathrm{IV}}$, as indeed an isovector operator. The relevant isospin selection rules and relations needed to evaluate isospinreduced two-body matrix elements of this operator are provided for reference in Appendix E.

\section{RELATIONS FOR BILINEAR OPERATORS}

The essential input for working with a two-body operator in a many-body calculation is its two-body matrix elements. As illustrated for the familiar case of the intrinsic kinetic energy in Sec. III, two main approaches exist for evaluating these matrix elements. These require reexpressing the operator, relative to its defining form in terms of $\mathbf{x}_{i}^{\prime}$ and $\mathbf{p}_{i}^{\prime}$, either: (1) as an explicit two-body operator, that is, expressed in terms of the two-body system's relative coordinate and momentum, or (2) decomposed into one-body and separable two-body terms. We estabish the necessary relations for the two-body operator obtained by transforming a bilinear operator to the intrinsic frame, first for an isoscalar bilinear operator (Sec. VA), then for an isovector bilinear operator (Sec. $\mathrm{VB}$ ).

\section{A. Isoscalar bilinear operators}

We now consider the problem of transforming a "bilinear" spherical tensor operator to the intrinsic frame. That is, we start with the one-body operator obtained from a spherical tensor product of the form $(\boldsymbol{\eta} \times \boldsymbol{\xi})_{L_{0}}$, where $\boldsymbol{\eta}$ and $\boldsymbol{\xi}$ represent either coordinate and/or momentum vectors or oscillator creation and/or annihilation operators. These latter (ladder) operators are defined and their properties reviewed in Appendix F. We are thus considering a one-body operator of the form

$$
T_{L_{0}}=U\left[(\boldsymbol{\eta} \times \boldsymbol{\xi})_{L_{0}}\right]=\sum_{i}\left(\boldsymbol{\eta}_{i} \times \boldsymbol{\xi}_{i}\right)_{L_{0}} .
$$

As the operators $\boldsymbol{\eta}$ and $\boldsymbol{\xi}$ are spherical tensor operators of rank 1, i.e., vector operators, their angular momenta can couple to give a total angular momentum $L_{0}=0,1$, or 2 . 
All such bilinear operators have the same structure, as far as their transformation to the intrinsic frame is concerned. We can therefore treat all such operators generically in a single, generic derivation. This affords not only a certain efficiency of effort but also highlights the otherwise nonobvious parallels (and distinctions) among a variety of structurally similar operators. As already motivated in the introduction (Sec. II), depending on the nature of the basis for the problem, we may find it necessary to recast the bilinear operator into one of two forms: (1) a one body contribution plus a separable two-body operator or (2) a relative two-body operator.

Regardless of whether $\boldsymbol{\eta}$ and $\boldsymbol{\xi}$ represent $\mathbf{x}, \mathbf{k}, \mathbf{c}^{\dagger}$, or $\mathbf{c}$, the transformation to the intrinsic frame, in either (17) for the coordinates and momenta or $(\mathrm{F} 13)$ for the ladder operators, is of the form:

$$
\boldsymbol{\eta}_{i}^{\prime}=\boldsymbol{\eta}_{i}-\frac{1}{A} \sum_{j} \boldsymbol{\eta}_{j} \quad \boldsymbol{\xi}_{i}^{\prime}=\boldsymbol{\xi}_{i}-\frac{1}{A} \sum_{j} \boldsymbol{\xi}_{j}
$$

The intrinsic operator $T_{L_{0}}^{\prime}$ obtained from $T_{L_{0}}$, is then defined by

$$
T_{L_{0}}^{\prime}=\sum_{i}\left(\boldsymbol{\eta}_{i}^{\prime} \times \boldsymbol{\xi}_{i}^{\prime}\right)_{L_{0}}
$$

and thus has the form

$$
T_{L_{0}}^{\prime}=\sum_{i}\left[\left(\boldsymbol{\eta}_{i}-\frac{1}{A} \sum_{j} \boldsymbol{\eta}_{j}\right) \times\left(\boldsymbol{\xi}_{i}-\frac{1}{A} \sum_{k} \boldsymbol{\xi}_{k}\right)\right]_{L_{0}} .
$$

When multiplied out, this expression for the intrinsic operator involves double and even triple sums over particle indices:

$$
T_{L_{0}}^{\prime}=\sum_{i}\left(\boldsymbol{\eta}_{i} \times \boldsymbol{\xi}_{i}\right)_{L_{0}}-\frac{1}{A} \sum_{i k}\left(\boldsymbol{\eta}_{i} \times \boldsymbol{\xi}_{k}\right)_{L_{0}}-\frac{1}{A} \sum_{i j}\left(\boldsymbol{\eta}_{j} \times \boldsymbol{\xi}_{i}\right)_{L_{0}}+\frac{1}{A^{2}} \sum_{i j k}\left(\boldsymbol{\eta}_{j} \times \boldsymbol{\xi}_{k}\right)_{L_{0}} .
$$

The intrinsic operator is no longer purely a one-body operator. However, this expression involves at most two-body, not three-body, contributions: the triple sum $\propto \sum_{i j k}\left(\boldsymbol{\eta}_{j} \times \boldsymbol{\xi}_{k}\right)_{L_{0}}$ in the final term involves summation over an index $i$ not appearing in the summand, which simply yields a counting factor (recall $\sum_{i} 1=A$ ).

First, we examine the structure of the expression in 40 . Renaming summation indices (and recognizing $\sum_{i} 1=A$ ) allows us to collect like terms, leaving only two distinct terms:

$$
T_{L_{0}}^{\prime}=\sum_{i}\left(\boldsymbol{\eta}_{i} \times \boldsymbol{\xi}_{i}\right)_{L_{0}}-\frac{1}{A} \sum_{i j}\left(\boldsymbol{\eta}_{i} \times \boldsymbol{\xi}_{j}\right)_{L_{0}}
$$

The first term we recognize as simply the original, uncorrected one-body operator $T_{L_{0}}$, while the remaining term can be factored into parts which act only on the center-of-mass degree of freedom:

$$
\underbrace{T_{L_{0}}^{\prime}}_{\text {Intrinsic }}=\underbrace{\sum_{i}\left(\boldsymbol{\eta}_{i} \times \boldsymbol{\xi}_{i}\right)_{L_{0}}}_{\text {One-body }}-\underbrace{\frac{1}{A}\left[\left(\sum_{i} \boldsymbol{\eta}_{i}\right) \times\left(\sum_{j} \boldsymbol{\xi}_{j}\right)\right]_{L_{0}}}_{\text {Center-of-mass }} .
$$

Thus, as with the dipole operator in (29), the intrinsic operator conveniently separates into the "naive" one-body operator and a c.m. recoil contribution. 
The practical significance of this separation 42 similarly arises when we set out to evaluate matrix elements of the intrinsic operator between wave functions obtained from solving the many-body problem, e.g., for transition matrix elements, moments, or expectation values. Again, for NCCI calculations in the traditional $N_{\text {max }}$-truncated oscillator basis, the c.m. motion factorizes and is described by an oscillator $0 s$ wave function.

For the case where the bilinear operator is nonscalar $\left(L_{0}>0\right)$, the c.m. contribution $\propto\left\langle 0 s\left\|\left(\boldsymbol{\eta}_{\text {c.m. }} \times \boldsymbol{\xi}_{\text {c.m. }}\right)_{L_{0}}\right\| 0 s\right\rangle$ vanishes identically, by the angular momentum triangle selection rule. Then matrix elements of the naive one-body operator may again be evaluated in place of matrix elements of the more computationally demanding two-body intrinsic operator. Thus, e.g., the naive one-body quadrupole operator is commonly used in place of the intrinsic quadrupole operator in $N_{\text {max }}$-truncated oscillator-basis NCCI calculations. (So far we have strictly only justified this for the isoscalar, or mass, quadrupole operator, while the less obvious case of the electric quadrupole operator is addressed below in Sec. VB.) However, this substitution is no longer justified when other bases are used and the factorized $0 s$ c.m. wave function is sacrificed.

For a scalar bilinear operator $\left(L_{0}=0\right)$, no such angular momentum selection rule applies, and the c.m. correction to a matrix element is in general nonzero. Thus, e.g., in evaluating the r.m.s. radius, it is necessary to calculate the expectation value of the intrinsic squared radius operator, which is a rotational scalar. The expectation value of the naive one-body operator does not simply equal that of the intrinsic operator. Nonetheless, the c.m. correction reduces to the expectation value of $r_{\mathrm{c} . \mathrm{m} .}^{2}=\mathbf{x}_{\mathrm{c} . \mathrm{m} .} \cdot \mathbf{x}_{\mathrm{c} . \mathrm{m}}$. in a $0 s$ oscillator state, which is analytically known from simple application of harmonic oscillator laddering relations (e.g., Ref. [139]). Thus, when working in an $N_{\max }$-truncated oscillator basis, the one-body operator can be used in place of the intrinsic operator by application of a simple correction. For other bases, the full two-body intrinsic operator must, in general, be used.

Next, we pursue the representation of $T_{L_{0}}^{\prime}$ as a one-body contribution plus a separable two-body operator. Although the expression (41) involves both single and double sums over particle indices, these terms do not directly represent the one-body and two-body parts. To bring the double sums into the form (8) required for a two-body operator, recall that we must extract the diagonal terms of the sum [via [C3] ], leaving behind a restricted sum $\left(\sum_{i j}^{\prime}\right)$, and we must also ensure that the summand is symmetrized with respect to interchange of particle indices $(i \leftrightarrow j)$ :

$$
T_{L_{0}}^{\prime}=\left(1-\frac{1}{A}\right) \sum_{i}\left(\boldsymbol{\eta}_{i} \times \boldsymbol{\xi}_{i}\right)_{L_{0}}-\frac{1}{2 A} \sum_{i j}^{\prime}\left[\left(\boldsymbol{\eta}_{i} \times \boldsymbol{\xi}_{j}\right)_{L_{0}}+\left(\boldsymbol{\eta}_{j} \times \boldsymbol{\xi}_{i}\right)_{L_{0}}\right] .
$$

We recognize the first and second terms in this expression (43) as representing one-body and two-body operators, respectively. Explicitly, in the notation of Sec. III A.

$$
T_{L_{0}}^{\prime}=\left(1-\frac{1}{A}\right) U\left[(\boldsymbol{\eta} \times \boldsymbol{\xi})_{L_{0}}\right]-\frac{1}{A} V\left[\left(\boldsymbol{\eta}_{1} \times \boldsymbol{\xi}_{2}\right)_{L_{0}}+\left(\boldsymbol{\eta}_{2} \times \boldsymbol{\xi}_{1}\right)_{L_{0}}\right] .
$$

Note that each term $\left(\boldsymbol{\eta}_{1} \times \boldsymbol{\xi}_{2}\right)_{L_{0}}$ or $\left(\boldsymbol{\eta}_{2} \times \boldsymbol{\xi}_{1}\right)_{L_{0}}$ in the two-body operator is the product of a factor acting only on the first particle and a factor acting only on the second particle. This separable form permits the use of Racah's reduction formula to evaluate two-body reduced matrix elements, in terms of the much more easily computed reduced matrix elements of $\boldsymbol{\eta}$ and $\boldsymbol{\xi}$ in the single-particle basis.

Alternatively, we recast $T_{L_{0}}^{\prime}$ as a relative two-body operator. To do so, recall from our treatment of the dipole operator (Sec. IV) we must now upgrade the one-body contribution 
so that it is represented as a two-body operator [via (C1)]:

$$
T_{L_{0}}^{\prime}=\frac{1}{2 A} \sum_{i j}^{\prime}\left[\left(\boldsymbol{\eta}_{i} \times \boldsymbol{\xi}_{i}\right)_{L_{0}}+\left(\boldsymbol{\eta}_{j} \times \boldsymbol{\xi}_{j}\right)_{L_{0}}\right]-\frac{1}{2 A} \sum_{i j}^{\prime}\left[\left(\boldsymbol{\eta}_{i} \times \boldsymbol{\xi}_{j}\right)_{L_{0}}+\left(\boldsymbol{\eta}_{j} \times \boldsymbol{\xi}_{i}\right)_{L_{0}}\right] \text {. }
$$

While each term here is symmetric under interchange of particle indices, it is still not immediately apparent that the two-body operator involves only the relative coordinate degree of freedom. We must refactor (45) to obtain

$$
T_{L_{0}}^{\prime}=\frac{1}{2 A} \sum_{i j}^{\prime}\left[\left(\boldsymbol{\eta}_{i}-\boldsymbol{\eta}_{j}\right) \times\left(\boldsymbol{\xi}_{i}-\boldsymbol{\xi}_{j}\right)\right]_{L_{0}} .
$$

Explicitly recognizing this expression as representing a relative two-body operator, in the notation of (8), we have

$$
T_{L_{0}}^{\prime}=\frac{2}{A} V[\underbrace{\frac{1}{2}\left[\left(\boldsymbol{\eta}_{1}-\boldsymbol{\eta}_{2}\right) \times\left(\boldsymbol{\xi}_{1}-\boldsymbol{\xi}_{2}\right)\right]_{L_{0}}}_{T_{\mathrm{rel}, L_{0}}}]
$$

As with the dipole operator in (33), we have extracted the $A$ dependence from the definition of the two-body operator $V[\cdots]$ appearing in (47), and have extracted an additional factor of 2 to ensure that the intrinsic operator $T_{L_{0}}^{\prime}$ simply reduces to the relative operator

$$
T_{\text {rel }, L_{0}}=\frac{1}{2}\left[\left(\boldsymbol{\eta}_{1}-\boldsymbol{\eta}_{2}\right) \times\left(\boldsymbol{\xi}_{1}-\boldsymbol{\xi}_{2}\right)\right]_{L_{0}}
$$

appearing inside the $V[\cdots]$ when evaluated on the two-particle space. We need only evaluate the $A$-independent matrix elements of this relative operator on the two-particle space. Then, as usual, matrix elements of $V\left[T_{\text {rel }, L_{0}}\right]$ on the many-particle space follow by second quantization. These must then be scaled by the counting factor $2 / A$ to yield matrix elements for the full intrinsic operator $T_{L_{0}}^{\prime}$.

\section{B. Isovector bilinear operators}

Electromagnetic operators of bilinear type are not of the simple isoscalar form (36) but rather distinguish protons and neutrons. As with the dipole operator in Sec. IV] rather than restricting the summation to just protons or just neutrons, which would sacrifice the simplicity of working with a true one-body operator, we instead modify the operator on the single-particle space so that it selects either for protons or for neutrons. Thus, we have proton and neutron bilinear operators

$$
\begin{aligned}
& T_{p, L_{0}}=\sum_{i}\left(\boldsymbol{\eta}_{i} \times \boldsymbol{\xi}_{i}\right)_{L_{0}} \delta_{i, p} \\
& T_{n, L_{0}}=\sum_{i}\left(\boldsymbol{\eta}_{i} \times \boldsymbol{\xi}_{i}\right)_{L_{0}} \delta_{i, n}
\end{aligned}
$$

where, again, $\delta_{i, p}=1$ if the $i$ th particle is a proton and $\delta_{i, p}=0$ if the $i$ th particle is a neutron, and vice versa for $\delta_{i, n}$. 
Although the one-body operator $T_{p, L_{0}}$ acts only upon the protons (and similarly $T_{n, L_{0}}$ acts only upon the neutrons), note that this is no longer true for the corresponding intrinsic operator. Taking, for example, the intrinsic proton operator

$$
T_{p, L_{0}}^{\prime}=\sum_{i}\left[\left(\boldsymbol{\eta}_{i}-\frac{1}{A} \sum_{j} \boldsymbol{\eta}_{j}\right) \times\left(\boldsymbol{\xi}_{i}-\frac{1}{A} \sum_{k} \boldsymbol{\xi}_{k}\right)\right]_{L_{0}} \delta_{i, p},
$$

neutrons contribute through the c.m. recoil corrections. That is, while the factor $\delta_{i, p}$ ensures that only values of the particle index $i$ which correspond to protons contribute, there is no such restriction on the values of the particle indices $j$ and $k$ which contribute ${ }^{6}$

As with the dipole operator in Sec. IV, it is convenient to consolidate the treatment of the proton and neutron bilinear operators into an isovector bilinear operator. We first use the selection operator $\delta_{\alpha}$, defined in $(23)$, to define

$$
T_{\alpha, L_{0}}=\sum_{i}\left(\boldsymbol{\eta}_{i} \times \boldsymbol{\xi}_{i}\right)_{L_{0}} \delta_{i, \alpha}
$$

where the proton operator is obtained for $\alpha=+1$, and the neutron operator for $\alpha=-1$. The proton or neutron operator then separates into isoscalar and isovector parts as

$$
T_{\alpha, L_{0}}=\frac{1}{2} \underbrace{\left[\sum_{i}\left(\boldsymbol{\eta}_{i} \times \boldsymbol{\xi}_{i}\right)_{L_{0}}\right]}_{T_{L_{0}}}+\alpha \frac{1}{2} \underbrace{\left[\sum_{i}\left(\boldsymbol{\eta}_{i} \times \boldsymbol{\xi}_{i}\right)_{L_{0}} \tau_{i 0}\right]}_{T_{\mathrm{IV}, L_{0}}} .
$$

The first term simply involves the isoscalar operator $T_{L_{0}}$ of $(36)$, while the second term defines the isovector operator

$$
T_{\mathrm{IV}, L_{0}}=\sum_{i}\left(\boldsymbol{\eta}_{i} \times \boldsymbol{\xi}_{i}\right)_{L_{0}} \tau_{i 0}
$$

The intrinsic form of this isovector bilinear operator, obtained by the substitution (37), is

$$
T_{\mathrm{IV}, L_{0}}^{\prime}=\sum_{i}\left[\left(\boldsymbol{\eta}_{i}-\frac{1}{A} \sum_{j} \boldsymbol{\eta}_{j}\right) \times\left(\boldsymbol{\xi}_{i}-\frac{1}{A} \sum_{k} \boldsymbol{\xi}_{k}\right)\right]_{L_{0}} \tau_{i 0} .
$$

Multiplying out the product again yields terms involving summations over one or more particle indices, as in (40), but now also involving the isospin factor of $\tau_{i 0}$ :

$$
\begin{aligned}
T_{\mathrm{IV}, L_{0}}^{\prime}=\sum_{i}\left(\boldsymbol{\eta}_{i} \times \boldsymbol{\xi}_{i}\right)_{L_{0}} \tau_{i 0} & \\
-\frac{1}{A} \sum_{i k}\left(\left(\boldsymbol{\eta}_{i} \tau_{i 0}\right) \times \boldsymbol{\xi}_{k}\right)_{L_{0}}-\frac{1}{A} \sum_{i j}\left(\boldsymbol{\eta}_{j} \times\left(\boldsymbol{\xi}_{i} \tau_{i 0}\right)\right)_{L_{0}} & +\frac{1}{A^{2}} \sum_{i j k} \tau_{i 0}\left(\boldsymbol{\eta}_{j} \times \boldsymbol{\xi}_{k}\right)_{L_{0}} .
\end{aligned}
$$

\footnotetext{
${ }^{6}$ The proton and neutron intrinsic bilinear operators are thus substantially different in nature from protononly or neutron-only interaction two-body operators, most notably the Coulomb interaction, which genuinely only acts on protons. These operators have a different isospin structure, involving not only isoscalar and isovector but also isotensor contributions.
} 
The operator $\tau_{i 0}$, although isovector, is a rotational scalar, and may thus be moved around within the products without regard for the angular momentum coulpling: here, in (55), we group it with the other factors involving the particle index $i$, to emphasize this connection. In the triple sum appearing in the final term, $\propto \sum_{i j k} \tau_{i 0}\left(\boldsymbol{\eta}_{j} \times \boldsymbol{\xi}_{k}\right)_{L_{0}}$, the summation over $i$ simply yields the counting factor $\sum_{i} \tau_{i 0}=Z-N$, as in (30).

However, the factors of $\tau_{i 0}$ mean that, even after renaming particle indices, there are no longer any strictly identical terms to combine as in (41). Rather, we can arrange the expression into three sums involving different combinations of summation indices and $\tau_{i 0}$ dependences:

$$
\begin{aligned}
T_{\mathrm{IV}, L_{0}}^{\prime}=\sum_{i}\left(\boldsymbol{\eta}_{i} \times \boldsymbol{\xi}_{i}\right)_{L_{0}} \tau_{i 0} & \\
-\frac{1}{A} \sum_{i j}\left[\left(\left(\boldsymbol{\eta}_{i} \tau_{i 0}\right) \times \boldsymbol{\xi}_{j}\right)_{L_{0}}+\left(\boldsymbol{\eta}_{j} \times\left(\boldsymbol{\xi}_{i} \tau_{i 0}\right)\right)_{L_{0}}\right] & +\frac{1}{A}\left(\frac{Z-N}{A}\right) \sum_{i j}\left(\boldsymbol{\eta}_{i} \times \boldsymbol{\xi}_{j}\right)_{L_{0}} .
\end{aligned}
$$

The first term of $(56)$ is recognized as the naive one-body operator $T_{\mathrm{IV}, L_{0}}$ of $(53)$. The final term is again purely a c.m. term $\propto\left(\boldsymbol{\eta}_{\text {c.m. }} \times \boldsymbol{\xi}_{\text {c.m. }}\right)_{L_{0}}$. However, the isospin factors in the middle terms of (56) preclude the separation of $T_{\mathrm{IV}, L_{0}}^{\prime}$ into one-body and c.m. terms, in the simple way obtained above for the isoscalar bilinear operator in the corresponding expression (42). The question thus arises as to whether or not, when the c.m. wave function has known $0 s$ form, the naive one-body operator can still be used in place of the full intrinsic operator - and, if not, what is the expression for the c.m. correction?

The middle terms of (56), involving $\sum_{i j}\left(\left(\boldsymbol{\eta}_{i} \tau_{i 0}\right) \times \boldsymbol{\xi}_{j}\right)_{L_{0}}$ and $\sum_{i j}\left(\boldsymbol{\eta}_{j} \times\left(\boldsymbol{\xi}_{i} \tau_{i 0}\right)\right)_{L_{0}}$ can most simply be addressed by leveraging the results for the dipole operator obtained in Sec. IV to represent them in terms of intrinsic and c.m. operators to which selection rules can be applied. While the dipole operator was defined in Sec. IV in terms of the spatial coordinate $\mathbf{x}$, we may generalize this definition to a general coordinate, momentum, or ladder operator $\boldsymbol{\xi}$, letting $\mathbf{D}^{\boldsymbol{\xi}}=\sum_{i} \boldsymbol{\xi}_{i}$, analogous to 19 , and $\mathbf{D}_{\mathrm{IV}}^{\boldsymbol{\xi}}=\sum_{i} \boldsymbol{\xi}_{i} \tau_{i 0}$, analogous to (25). Then, $\mathbf{D}^{\xi}$ is again a pure c.m. operator, and we have the relation $\mathbf{D}_{\mathrm{IV}}^{\xi \prime}=\mathbf{D}_{\mathrm{IV}}^{\xi}-[(Z-N) / A] \mathbf{D}^{\xi}$, analogous to (30). Thus, taking the first of the middle terms in (56), we recognize

$$
\sum_{i j}\left(\left(\boldsymbol{\eta}_{i} \tau_{i 0}\right) \times \boldsymbol{\xi}_{j}\right)_{L_{0}}=\left(\mathbf{D}_{\mathrm{IV}}^{\eta} \times \mathbf{D}^{\boldsymbol{\xi}}\right)_{L_{0}}=\left(\mathbf{D}_{\mathrm{IV}}^{\eta \prime} \times \mathbf{D}^{\boldsymbol{\xi}}\right)_{L_{0}}+\left(\frac{Z-N}{A}\right)\left(\mathbf{D}^{\boldsymbol{\eta}} \times \mathbf{D}^{\boldsymbol{\xi}}\right)_{L_{0}} .
$$

The matrix element of the first term in this expression vanishes by the angular momentum triangle selection rule, which enforces vanishing $\left\langle 0 s\left\|\mathbf{D}^{\xi}\right\| 0 s\right\rangle$, while the second term of this expression is a pure c.m. operator of the same form as the last term of (56). The second of the middle terms in (56) may be handled similarly. Thus, the matrix element of $T_{\mathrm{IV}, L_{0}}^{\prime}$ between states with $0 s$ center-of-mass motion again separates into the naive one-body contribution plus a pure center-of-mass contribution, which again vanishes by angular momentum selection if $L_{0} \neq 0$.

Keeping in mind our first aim, to separate $T_{\mathrm{IV}, L_{0}}^{\prime}$ into one-body and separable two-body parts, we extract the diagonal terms from the double sums in (56) [via (C3)], to obtain the desired restricted sum $\left(\sum_{i j}^{\prime}\right)$, and explicitly symmetrize the expressions under interchange 
of particle indices $(i \leftrightarrow j)$, obtaining

$$
\begin{aligned}
T_{\mathrm{IV}, L_{0}}^{\prime}=\left(1-\frac{2}{A}\right) \sum_{i}\left(\boldsymbol{\eta}_{i} \times \boldsymbol{\xi}_{i}\right)_{L_{0}} \tau_{i 0} & +\frac{1}{A}\left(\frac{Z-N}{A}\right) \sum_{i}\left(\boldsymbol{\eta}_{i} \times \boldsymbol{\xi}_{i}\right)_{L_{0}} \\
-\frac{1}{2 A} \sum_{i j}^{\prime}\left[\left(\boldsymbol{\eta}_{i} \times \boldsymbol{\xi}_{j}\right)_{L_{0}}+\left(\boldsymbol{\eta}_{j} \times \boldsymbol{\xi}_{i}\right)_{L_{0}}\right]\left(\tau_{i 0}+\tau_{j 0}\right) & \\
& +\frac{1}{2 A}\left(\frac{Z-N}{A}\right) \sum_{i j}^{\prime}\left[\left(\boldsymbol{\eta}_{i} \times \boldsymbol{\xi}_{j}\right)_{L_{0}}+\left(\boldsymbol{\eta}_{j} \times \boldsymbol{\xi}_{i}\right)_{L_{0}}\right] .
\end{aligned}
$$

Explicitly recognizing the first two terms as one-body operators and the latter two terms as two-body operators, we have

$$
\begin{aligned}
T_{\mathrm{IV}, L_{0}}^{\prime}=\left(1-\frac{2}{A}\right) U[ & \left.(\boldsymbol{\eta} \times \boldsymbol{\xi})_{L_{0}} \tau_{0}\right]+\frac{1}{A}\left(\frac{Z-N}{A}\right) U\left[(\boldsymbol{\eta} \times \boldsymbol{\xi})_{L_{0}}\right] \\
& -\frac{1}{A} V\left[\left[\left(\boldsymbol{\eta}_{1} \times \boldsymbol{\xi}_{2}\right)_{L_{0}}+\left(\boldsymbol{\eta}_{2} \times \boldsymbol{\xi}_{1}\right)_{L_{0}}\right]\left(\tau_{10}+\tau_{20}\right)\right] \\
& -\frac{1}{A}\left(\frac{Z-N}{A}\right) V\left[\left(\boldsymbol{\eta}_{1} \times \boldsymbol{\xi}_{2}\right)_{L_{0}}+\left(\boldsymbol{\eta}_{2} \times \boldsymbol{\xi}_{1}\right)_{L_{0}}\right] .
\end{aligned}
$$

The second and fourth terms in (59) involve the one-body and separable two-body operators which already appear in the analogous decomposition (44) for the isoscalar operator $T_{L_{0}}^{\prime}$, although now with different, $(Z-N)$-dependent coefficients. The first and third terms involve new, isovector one-body and two-body operators. Note that $\boldsymbol{\tau}_{1}+\boldsymbol{\tau}_{2}=2 \mathrm{~T}$ is simply proportional to the total isospin operator $\mathbf{T}$ for the two-body system, so the combination $\tau_{10}+\tau_{20}$ appearing in the third term of $(59)$ is simply $2 T_{0}$.

Alternatively, to recast $T_{\mathrm{IV}, L_{0}}^{\prime}$ as a relative two-body operator, we must upgrade the one-body contribution in (58) so that it is represented as a two-body operator [via (C1)]. However, the algebra is considerably more involved than for the isoscalar operator $T_{L_{0}}^{\prime}$ in (45) above, and we omit the details. [We only note that, in order to combine like terms, it is helpful to be able to introduce or remove an explicit dependence on the isospin operators $\tau_{i 0}$ and $\tau_{j 0}$ within the sums, via the identity $(Z-N) \sum_{i j}^{\prime}\left(u_{i}+u_{j}\right)=\sum_{i j}^{\prime}\left(u_{i} \tau_{i 0}+u_{j} \tau_{j 0}\right)+$ $(A-1) \sum_{i j}^{\prime}\left(u_{i} \tau_{j 0}+u_{j} \tau_{i 0}\right)$, which may be obtained by combining (C1) with the identity $\sum_{i} \tau_{i 0}=Z-N$.] In the end, we obtain an expression for $T_{\mathrm{IV}, L_{0}}^{\prime}$ involving sums of the desired relative two-body form:

$$
\begin{aligned}
T_{\mathrm{IV}, L_{0}}^{\prime}=\frac{1}{2 A} \sum_{i j}^{\prime}\left[\left[\left(\boldsymbol{\eta}_{i}-\boldsymbol{\eta}_{j}\right) \times\left(\boldsymbol{\xi}_{i}-\boldsymbol{\xi}_{j}\right)\right]_{L_{0}}\left(\tau_{i 0}+\tau_{j 0}\right)\right] \\
-\frac{1}{2 A}\left(\frac{Z-N}{A}\right) \sum_{i j}^{\prime}\left[\left(\boldsymbol{\eta}_{i}-\boldsymbol{\eta}_{j}\right) \times\left(\boldsymbol{\xi}_{i}-\boldsymbol{\xi}_{j}\right)\right]_{L_{0}} .
\end{aligned}
$$


Explicitly recognizing both sums in (60) as two-body operators, we have

$$
\begin{aligned}
& T_{\mathrm{IV}, L_{0}}^{\prime}=\frac{2}{A} V[\underbrace{\overbrace{\frac{1}{2\left[\left(\boldsymbol{\eta}_{1}-\boldsymbol{\eta}_{2}\right) \times\left(\boldsymbol{\xi}_{1}-\boldsymbol{\xi}_{2}\right)\right]_{L_{0}}}}^{T_{\mathrm{rel}, L_{0}}}\left(2 T_{0}\right)}_{T_{\mathrm{IV}, \mathrm{rel}, L_{0}}}] \\
& -\frac{2}{A}\left(\frac{Z-N}{A}\right) V[\underbrace{\frac{1}{2}\left[\left(\boldsymbol{\eta}_{1}-\boldsymbol{\eta}_{2}\right) \times\left(\boldsymbol{\xi}_{1}-\boldsymbol{\xi}_{2}\right)\right]_{L_{0}}}_{T_{\mathrm{rel}, L_{0}}}] .
\end{aligned}
$$

Here we recognize that, as noted above, $\boldsymbol{\tau}_{1}+\boldsymbol{\tau}_{2}=\boldsymbol{\tau} \equiv 2 \mathrm{~T}$ is simply proportional to the total isospin operator on the two-particle space, and so $\tau_{10}+\tau_{20}=2 T_{0}$.

Both two-body operators appearing in (61) are manifestly Galilean-invariant (and thus relative) two-body operators. They are expressed in terms of the relative operators $T_{\mathrm{IV}, \mathrm{rel}, L_{0}}=2 T_{\mathrm{rel}, L_{0}} T_{0}$ and $T_{\mathrm{rel}, L_{0}}$ on the two-particle space, where $T_{\mathrm{rel}, L_{0}}$ was defined previously in (48). Note that, in both terms of (61), as previously in (47), we extract a factor of 2 in the overall coefficient, so that the intrinsic operator, when evaluated on the two-body system $(A=2)$, is more simply related to the relative operators defined on the two-particle space.

To set up a many-body calculation, we need only evaluate the $A$-independent and $(Z-N)$ independent matrix elements of these two relative operators on the two-particle space. These two-body matrix element may thus be evaluated once and for all, independent of the number of nucleons in the system. Then the two-body matrix elements for the full intrinsic operator $T_{\mathrm{IV}, L_{0}}^{\prime}$ on a system with a given number of nucleons may then be obtained as the linear combination with the appropriate scale factors, involving $A$ and $Z-N$, from (61). The twobody matrix elements of $T_{\mathrm{rel}, L_{0}}$ have already been noted as being required for the isoscalar operator $T_{L_{0}}^{\prime}$, via (47). Furthermore, the two-body matrix elements of $T_{\mathrm{IV}, \mathrm{rel}, L_{0}}$ may be simply obtained from those of $T_{\mathrm{rel}, L_{0}}$, as given in isospin scheme in (E1), due to the clean factorization of $T_{\mathrm{IV}, \mathrm{rel}, L_{0}}$ into spatial and isospin factors.

With the isoscalar and isovector bilinear operators now addressed, we can, of course, recover the intrinsic form of the proton or neutron bilinear operators. We recall the expression (52) for $T_{\alpha, L_{0}}$ in terms of $T_{L_{0}}$ and $T_{\mathrm{IV}, L_{0}}$ and combine the intrinsic forms $T_{L_{0}}^{\prime}$ from (47) and $T_{\mathrm{IV}, L_{0}}^{\prime}$ from 61 of these operators, to obtain

$$
\begin{aligned}
T_{\alpha, L_{0}}^{\prime}=\frac{1}{A}\left(1-\alpha \frac{Z-N}{A}\right) V\left[\frac{1}{2}\left[\left(\boldsymbol{\eta}_{1}-\boldsymbol{\eta}_{2}\right) \times\left(\boldsymbol{\xi}_{1}-\boldsymbol{\xi}_{2}\right)\right]_{L_{0}}\right] \\
+\alpha \frac{1}{A} V\left[\frac{1}{2}\left[\left(\boldsymbol{\eta}_{1}-\boldsymbol{\eta}_{2}\right) \times\left(\boldsymbol{\xi}_{1}-\boldsymbol{\xi}_{2}\right)\right]_{L_{0}}\left(2 T_{0}\right)\right] .
\end{aligned}
$$

\section{CONCLUSION}

When solving the nuclear many-body problem, there is a tension between the simultaneous needs to respect antisymmetry under particle exchange and to preserve the underlying Galilean invariance of the problem. Typically, for computational methods which can be applied beyond the very lightest few-nucleon systems, manifest Galilean invariance is sacrificed in favor of manifest antisymmetry. However, we still require the ability to compute observables which are free of center-of-mass contamination. The consequence is that we must work with intrinsic operators for observables. 
In this work, we have derived generic expressions relating one-body operators which are bilinear in coordinates and/or momenta to their intrinsic counterparts. We give expressions directly usable for calculating two-body matrix elements in the laboratory frame, as well as matrix elements in the relative coordinate degree of freedom (which yield two-body matrix elements via the Moshinsky transformation). These formulae are applicable to both scalar and non-scalar bilinear operators, as well as to both the isoscalar and isovector forms of these operators. In appendices, we provide further results for reference in working with and evaluating two-body matrix elements of intrinsic operators, including the coefficients necessary to realize physical operators of interest in terms of the generic expressions for bilinear operators.

The derivations of the present expressions, while elementary in their methods, require care in their execution, in order to be of practical use to nuclear many-body practitioners. Our effort to synthesize and explicitly establish these results is motivated by their expected usefulness in implementing ab initio nuclear many-body calculations, using both traditional and $\operatorname{Sp}(3, \mathbb{R}) \supset \mathrm{U}(3)$ symmetry-adapted techniques.

\section{ACKNOWLEDGMENTS}

We thank Pieter Maris and James P. Vary for valuable discussions and Jakub Herko for a careful reading and detailed comments on the manuscript. This material is based upon work supported by the U.S. Department of Energy, Office of Science, Office of Nuclear Physics, under Award Number DE-FG02-95ER-40934. TRIUMF receives federal funding via a contribution agreement with the National Research Council of Canada.

\section{Appendix A: Physical operators realized as bilinear operators}

We have thus far kept our expression for the bilinear operators in the generic form $(\boldsymbol{\eta} \times$ $\boldsymbol{\xi})_{L_{0}}$, in both its isoscalar $(36)$ and isovector (53) variants. This generic form must then, of course, be specialized to the particular operators, representing either physical observables or group generators, of interest in nuclear physics applications. Once one of these operators $O$ is related, as

$$
O=\kappa U\left[(\boldsymbol{\eta} \times \boldsymbol{\xi})_{L_{0}}\right]
$$

to the generic bilinear form, then the translation to an explicitly intrinsic operator (by replacement $\boldsymbol{\eta} \rightarrow \boldsymbol{\eta}^{\prime}$ and $\boldsymbol{\xi} \rightarrow \boldsymbol{\xi}^{\prime}$ ) and subsequent decompositions into either separable or relative two-body forms follow immediately from the results of Sec. V]

In this appendix, we set out specializations of (A1) to specific operators of physical interest and highlight some of the practical consequences of the relations from Sec. $\mathrm{V}$ for these operators. We consider bilinear operators first involving coordinates and momenta $(\mathbf{x} \times \mathbf{x})_{L_{0}},(\mathbf{k} \times \mathbf{k})_{L_{0}}$, and $(\mathbf{x} \times \mathbf{k})_{L_{0}}$ - and then involving oscillator ladder operators $\left(\mathbf{c}^{\dagger} \times \mathbf{c}\right)_{L_{0}},\left(\mathbf{c}^{\dagger} \times \mathbf{c}^{\dagger}\right)_{L_{0}}$, and $(\mathbf{c} \times \mathbf{c})_{L_{0}}$ - with $L_{0}=0,1,2$. The results are summarized in Table I. The isoscalar forms are provided in the table, while the corresponding proton or neutron forms may be obtained through the corresponding substitutions in (49).

Although the relative operator $T_{\text {rel, } L_{0}}$ on the two-body space, as defined in (48), may clearly be expressed in terms of relative quantities $\boldsymbol{\eta}_{\text {rel }} \propto \boldsymbol{\eta}_{1}-\boldsymbol{\eta}_{2}$ and $\boldsymbol{\xi}_{\text {rel }} \propto \boldsymbol{\xi}_{1}-\boldsymbol{\xi}_{2}$, the proportionality factor depends upon the particular operators $\boldsymbol{\eta}$ and $\boldsymbol{\xi}$ involved. We must 
TABLE I. Physical operators represented in terms of one-body bilinear operators $T_{L_{0}}$, together with the relative operator $T_{\text {rel }, L_{0}}$ entering into the two-body representation of the corresponding intrinsic operator $T_{L_{0}}^{\prime}$. Coefficients and variables are as defined in (A1) and (A2). Only the isoscalar forms of the operators are explicitly tabulated, and normalization conventions may vary (see text). The values for blank entries should be taken as identical to those immediately above.

\begin{tabular}{|c|c|c|c|c|c|c|c|}
\hline \multirow[b]{2}{*}{ Operator } & \multirow[b]{2}{*}{$\kappa$} & \multicolumn{3}{|c|}{$T_{L_{0}}$} & \multicolumn{3}{|c|}{$T_{\mathrm{rel}, L_{0}}$} \\
\hline & & $\eta$ & $\xi$ & $L_{0}$ & $s$ & $\boldsymbol{\eta}_{\mathrm{rel}}$ & $\xi_{\text {rel }}$ \\
\hline$\sum_{i} x_{i}^{2}$ & $-\sqrt{3}$ & $\mathbf{x}$ & $\mathbf{x}$ & 0 & $\{1 / 2\}$ & $\mathbf{x}_{\mathrm{rel}}$ & $\mathbf{x}_{\mathrm{rel}}$ \\
\hline$Q_{2}$ & $\sqrt{\frac{15}{8 \pi}}$ & & & 2 & & & \\
\hline$T$ & $-\sqrt{3} \frac{\hbar^{2}}{2 m}$ & k & k & 0 & $\left\{\begin{array}{l}2 \\
1\end{array}\right\}$ & $\mathbf{k}_{\mathrm{rel}}$ & $\mathbf{k}_{\mathrm{rel}}$ \\
\hline $\mathbf{L}$ & $-i \sqrt{2}$ & $\mathrm{x}$ & k & 1 & 1 & $\mathbf{x}_{\mathrm{rel}}$ & $\mathbf{k}_{\mathrm{rel}}$ \\
\hline$N$ & $-\sqrt{3}$ & $\mathbf{c}^{\dagger}$ & c & 0 & 1 & $\mathbf{c}_{\mathrm{rel}}^{\dagger}$ & $\mathbf{c}_{\mathrm{rel}}$ \\
\hline $\mathbf{L}\left[=C_{1}^{(11)}\right]$ & $-\sqrt{2}$ & & & 1 & & & \\
\hline $\mathcal{Q}_{2}\left[=\sqrt{3} C_{2}^{(11)}\right]$ & $\sqrt{6}$ & & & 2 & & & \\
\hline$A_{0}^{(20)}$ & $-\frac{1}{\sqrt{2}}$ & $\mathbf{c}^{\dagger}$ & $\mathbf{c}^{\dagger}$ & 0 & 1 & $\mathbf{c}_{\mathrm{rel}}^{\dagger}$ & $\mathbf{c}_{\mathrm{rel}}^{\dagger}$ \\
\hline$A_{2}^{(20)}$ & $+\frac{1}{\sqrt{2}}$ & & & 2 & & & \\
\hline$B_{0}^{(02)}$ & $-\frac{1}{\sqrt{2}}$ & c & c & 0 & 1 & $\mathbf{c}_{\mathrm{rel}}$ & $\mathbf{c}_{\mathrm{rel}}$ \\
\hline$B_{2}^{(02)}$ & $+\frac{1}{\sqrt{2}}$ & & & 2 & & & \\
\hline
\end{tabular}

therefore allow for a scale factor $s$ in

$$
T_{\text {rel }, L_{0}}=s\left(\boldsymbol{\eta}_{\text {rel }} \times \boldsymbol{\xi}_{\text {rel }}\right)_{L_{0}},
$$

where $s$ is specified explicitly for the various physical operators in Table I. This scale factor is practically important in the evaluation of two-body matrix elements for $T_{L_{0}}^{\prime}$, when these are obtained via (47) from the matrix elements of $T_{\text {rel, } L_{0}}$ on the two-body space. If $\boldsymbol{\eta}$ and $\boldsymbol{\xi}$ represent coordinates or momenta, then $s$ depends upon the choice of convention in (12), but, if these variables represent oscillator ladder operators [see (F10)], then $s$ is simply unity.

Throughout this work, in spherical tensor coupling notation, we take a vector operator $\mathbf{A}$ to indicate the corresponding rank-1 covariant spherical tensor operator $A_{1}$, e.g., $(\boldsymbol{\eta} \times \boldsymbol{\xi})_{L_{0}} \equiv$ $\left(\eta_{1} \times \xi_{1}\right)_{L_{0}}$. The spherical components of $A_{1}$ are given in terms of the Cartesian components, as usual, by [141]

$$
A_{1, \pm 1}=\mp \frac{1}{\sqrt{2}}\left(A_{x} \pm i A_{y}\right) \quad A_{1,0}=A_{z}
$$

\section{Operators of the form $(\mathbf{x} \times \mathbf{x})_{L_{0}}$}

For the coupled product of a spherical tensor operator with itself, couplings of odd rank vanish identically by the symmetry properties of the Clebsch-Gordan coefficients. Therefore, only the couplings with $L_{0}=0,2$ arise for $(\mathbf{x} \times \mathbf{x})_{L_{0}}$. The isovector (or, rather, proton-only) forms give rise to the electric monopole $\left(L_{0}=0\right)$ and quadrupole $\left(L_{0}=2\right)$ operators. 
For $L_{0}=0$, we obtain the squared radius operator, taken already for illustration in Sec. III. The r.m.s. point-nucleon radius relative to the origin is obtained as $r_{m}=\left[A^{-1}\left\langle\sum_{i} x_{i}^{2}\right\rangle\right]^{1 / 2}$, where $x_{i}^{2} \equiv \mathbf{x}_{i} \cdot \mathbf{x}_{i}$. For the one-body summed squared radius operator $]^{7}$ appearing inside the expectation value, we have

$$
\sum_{i} x_{i}^{2}=U\left[(-\sqrt{3})(\mathbf{x} \times \mathbf{x})_{0}\right]
$$

Here we make use of the relation $\boldsymbol{\eta} \cdot \boldsymbol{\xi}=-\sqrt{3}(\boldsymbol{\eta} \times \boldsymbol{\xi})_{0}$. between the spherical tensor product of rank 0 and the standard vector dot product. We thus obtain, for the summed squared radius operator, the identifications $\kappa \rightarrow-\sqrt{3}, \boldsymbol{\eta} \rightarrow \mathbf{x}, \boldsymbol{\xi} \rightarrow \mathbf{x}$, and $L_{0} \rightarrow 0$, as given in Table [1.

The r.m.s. point-nucleon radius relative to the center of mass, as noted in Sec. II, is obtained by instead using the corresponding intrinsic coordinate within the expectation value, $r_{m}^{\prime}=\left[A^{-1}\left\langle\sum_{i} x_{i}^{\prime 2}\right\rangle\right]^{1 / 2}$. The summed squared intrinsic coordinate operator within the expectation value may then be represented in separable form, by (44), as

$$
\begin{aligned}
\sum_{i} x_{i}^{\prime 2} & =\left(1-\frac{1}{A}\right) U\left[(-\sqrt{3})(\mathbf{x} \times \mathbf{x})_{0}\right]-\frac{1}{A} V\left[2(-\sqrt{3})\left(\mathbf{x}_{1} \times \mathbf{x}_{2}\right)_{0}\right] \\
& =\left(1-\frac{1}{A}\right) U\left[x^{2}\right]-\frac{1}{A} V\left[2 \mathbf{x}_{1} \cdot \mathbf{x}_{2}\right]
\end{aligned}
$$

where $x^{2} \equiv \mathbf{x} \cdot \mathbf{x}$, or in manifestly two-body form, by (47) and then $(12)$, as

$$
\begin{aligned}
\sum_{i} x_{i}^{\prime 2} & =\frac{2}{A} V\left[(-\sqrt{3}) \frac{1}{2}\left[\left(\mathbf{x}_{1}-\mathbf{x}_{2}\right) \times\left(\mathbf{x}_{1}-\mathbf{x}_{2}\right)\right]_{0}\right] \\
& =\frac{2}{A} V\left[\left\{\begin{array}{c}
1 / 2 \\
1
\end{array}\right\} x_{\mathrm{rel}}^{2}\right],
\end{aligned}
$$

where $x_{\text {rel }}^{2} \equiv \mathbf{x}_{\text {rel }} \cdot \mathbf{x}_{\text {rel }}$. Similarly, the expectation value of the squared radius in the intrinsic frame may be represented in terms of the value calculated using the naive, one-body laboratory-frame operator, less a center-of-mass contribution, using (42):

$$
\left\langle\sum_{i} x_{i}^{\prime 2}\right\rangle=\left\langle\sum_{i} x_{i}^{2}\right\rangle-\left\{\begin{array}{c}
A \\
1
\end{array}\right\}\left\langle x_{\text {c.m. }}^{2}\right\rangle,
$$

where $x_{\text {c.m. }}^{2} \equiv \mathbf{x}_{\text {c.m. }} \cdot \mathbf{x}_{\text {c.m. }}$. This center-of-mass contribution is trivially known in the case of a factorized $0 s$ harmonic oscillator center-of-mass wave function.

The r.m.s. radius of the probability distribution of nucleons of a single species $\alpha$ (protons or neutrons), relative to the origin, is instead obtained as (see also Refs. [92, 94])

$$
\begin{aligned}
r_{\alpha} & =\left[\frac{1}{N_{\alpha}}\left\langle\sum_{i} x_{i}^{2} \delta_{i \alpha}\right\rangle\right]^{1 / 2} \\
& =\left[\frac{1}{N_{\alpha}}\left\langle U\left[(-\sqrt{3})(\mathbf{x} \times \mathbf{x})_{0} \delta_{\alpha}\right]\right\rangle\right]^{1 / 2} .
\end{aligned}
$$

\footnotetext{
7 While it might be tempting to denote the one-body summed squared radius operator by, say, $r^{2}$, this does not provide the basis for a robust notation, given likely confusion with the mean summed squared radius operator and the r.m.s. value itself.
} 
The operator appearing in the expectation value is thus now the proton-only or neutrononly version of the summed squared radius operator considered above and listed in Table I. and the results of Sec. $\mathrm{VB}$ now apply. The corresponding radius relative to the center of mass (of the nucleus as a whole, that is, not just of the nucleons of the same species) is obtained as the corresponding intrinsic operator. The intrinsic r.m.s. radius $r_{p}$ of the point proton distribution, in particular, is related, after hadronic physics corrections [142], to the experimentally accessible nuclear charge radius. This same proton instrinsic squared radius operator provides the leading order contribution to the E0 transition operator [143, 144].

In general, the intrinsic operator must be used in evaluating observables. However, for many-body calculations in which the wave function is known to factorize, with pure harmonic oscillator $0 s$ motion for the center of mass, the naive one-body squared radius or $E 0$ operator may be substituted for the intrinsic two-body operator, provided the known contribution arising from the zero-point motion of the center of mass is subtracted off. This may be determined by computing the $0 s$ expectation value of the center-of-mass terms in (42) or (56), as discussed in Sec. V.

For $L_{0}=2$, we obtain the quadrupole operator. The electromagnetic operator which induces $E 2$ transitions is, in the leading-order or impulse approximation, the proton-only operator with spherical components [104, 144]

$$
Q_{2, p}=\sum_{i} e_{i} x_{i}^{2} Y_{2}\left(\hat{\mathbf{x}}_{i}\right)
$$

where $Y_{2}$ denotes the spherical tensor with the spherical harmonics $Y_{2, \mu}$ as its components, the charge of the $i$ th nucleon is given by $e_{i}=e \delta_{i, p}$, and $\hat{\mathbf{x}}_{i}$ implicitly represents the polar angles for the $i$ th particle $\left(\mathbf{x}_{i}=x_{i} \hat{\mathbf{x}}_{i}\right)$. For purposes of identifying the factors in (A1) for Table II however, we need only consider the isoscalar (or mass) quadrupole operator, reflecting the combined proton and neutron point-nucleon density,

$$
Q_{2}=\sum_{i} x_{i}^{2} Y_{2}\left(\hat{\mathbf{x}}_{i}\right)
$$

in which we have omitted the electron charge factor $e$ as irrelevant. Noting the spherical tensor identity $x^{2} Y_{2}(\hat{\mathbf{x}})=[15 /(8 \pi)]^{1 / 2}(\mathbf{x} \times \mathbf{x})_{2}$, we thus have

$$
Q_{2}=U\left[\sqrt{\frac{15}{8 \pi}}(\mathbf{x} \times \mathbf{x})_{2}\right]
$$

giving the identifications $\kappa \rightarrow[15 /(8 \pi)]^{1 / 2}, \boldsymbol{\eta} \rightarrow \mathbf{x}, \boldsymbol{\xi} \rightarrow \mathbf{x}$, and $L_{0} \rightarrow 2$ in Table II The isoscalar and isovector forms may be combined as usual, by (52), to recover $Q_{2, p}$, which enters into electromagnetic observables, and $Q_{2, n}$, which enters into nuclear scattering observables [148].

\footnotetext{
${ }^{8}$ The operator $Q_{2, p}$ as defined in $\left(\mathrm{A} 9\right.$, and thus $Q_{2}$ as defined in $(\mathrm{A} 10)$, is normalized so as to match the $E 2$ transition operator as it naturally appears in the multipole expansion (e.g., Refs. [145-147]). The static quadrupole moment $Q$ is defined via the Cartesian quadrupole tensor $Q_{r s}=3 x_{r} x_{s}-x^{2} \delta_{r s}$ as the expectation value of $\sum_{i} Q_{z z, i}$ in the stretched state $|J J\rangle$, giving $Q=(16 \pi / 5)^{1 / 2}\left\langle J J\left|Q_{2,0}\right| J J\right\rangle$. Alternatively, the quadrupole operator may be normalized (e.g., Ref. [144]) as $Q_{2}^{\operatorname{mom}}=(16 \pi / 5)^{1 / 2} x^{2} Y_{2}(\hat{\mathbf{x}})=\sqrt{6}(\mathbf{x} \times \mathbf{x})_{2}$, so as to directly give the quadrupole moment, yielding instead $\kappa \rightarrow \sqrt{6}$. The latter normalization is consistent with that of the $\mathrm{SU}(3)$ quadrupole generator $\mathcal{Q}_{2}$ defined below [see $(\mathrm{A} 23)$ and $\left.(\mathrm{A} 24)\right]$.
} 
Again, the intrinsic operator must be used in evaluating observables. For many-body calculations in which the wave function is known to factorize, with pure harmonic oscillator $0 s$ motion for the center of mass, the naive one-body quadrupole operator may be used in the evaluation of quadrupole moments or $E 2$ transition matrix elements, since the matrix elements of the center-of-mass contributions vanish by angular momentum selection rules. This property is manifest for the isoscalar operator, from its simple separation into intrinsic and center-of-mass quadrupole operators as

$$
Q_{2}^{\prime}=Q_{2}-\left\{\begin{array}{c}
A \\
1
\end{array}\right\} Q_{2, \text { c.m. }}
$$

by (42). For the isovector quadrupole operator, the corresponding property follows from more detailed term-by-term analysis of (56), as discussed in Sec. VB.

\section{Operators of the form $(\mathbf{k} \times \mathbf{k})_{L_{0}}$}

For bilinear operators of the form $(\mathbf{k} \times \mathbf{k})_{L_{0}}$, again only the couplings with $L_{0}=0,2$ arise, while the coupling with $L_{0}=1$ vanishes identically by the symmetry properties of the Clebsch-Gordan coefficients. The summed squared momentum operator obtained for $L_{0}=0$ enters into the familiar kinetic energy operator $T$, taken already for illustration in Sec. II in the context of the intrinsic Hamiltonian. Since the expressions obtained for this operator closely match those for the summed squared radius operator above (Sec. A 1), it suffices to quote the results in Table I.

The explicit two-body expression for $T^{\prime}$, from (47), is

$$
T^{\prime}=\frac{2}{A} V[\underbrace{\frac{1}{2} \frac{\hbar^{2}}{2 m}\left[\left(\mathbf{k}_{1}-\mathbf{k}_{2}\right) \cdot\left(\mathbf{k}_{1}-\mathbf{k}_{2}\right)\right]}_{T_{\mathrm{rel}}}],
$$

where, in terms of the relative coordinate,

$$
T_{\text {rel }}=\frac{\hbar^{2}}{2 m}\left\{\begin{array}{l}
2 \\
1
\end{array}\right\} \mathbf{k}_{\mathrm{rel}} \cdot \mathbf{k}_{\mathrm{rel}} .
$$

Then, we note the one-body plus separable two-body form of the intrinsic kinetic energy obtained from (44) 9

$$
T^{\prime}=\frac{\hbar^{2}}{2 m}\left(1-\frac{1}{A}\right) U\left[k^{2}\right]-\frac{\hbar^{2}}{2 m} \frac{1}{A} V\left[2 \mathbf{k}_{1} \cdot \mathbf{k}_{2}\right],
$$

which may be used to evaluate the two-body matrix elements of $T^{\prime}$ in terms of one-body matrix elements of $\mathbf{k}$ and $k^{2}$.

Incidentally, the alternative prefactors in braces in (A14), arising as the factor $s$ from (A2), may be interpreted as introducing the reduced mass in the relative kinetic energy, as can be seen when the preceding expression is rearranged as

$$
T_{\text {rel }}=\frac{\hbar^{2}}{2\{m / 2, m\}} \mathbf{k}_{\text {rel }} \cdot \mathbf{k}_{\text {rel }} \text {. }
$$

${ }^{9}$ The comparative clarity of notation afforded by the one-body and two-body operator conventions of Sec. III A may be noted by comparison of A15 to prior expressions (see, e.g., Appendix A of Ref. 93]). 
The reduced mass is thus $m / 2$ under the mechanics convention for the relative coordinate but is simply the ordinary mass under the symmetric convention - this distinction in turn relates to different values for the relative oscillator length $b_{\text {rel }}$ arising below in (F14) under these two conventions.

\section{Operators of the form $(\mathbf{x} \times \mathbf{k})_{L_{0}}$}

All couplings $L_{0}=0,1,2$ are possible for $(\mathbf{x} \times \mathbf{k})_{L_{0}}$ or $(\mathbf{k} \times \mathbf{x})_{L_{0}}$. These two operators $(\mathbf{x} \times \mathbf{k})_{L_{0}}$ and $(\mathbf{k} \times \mathbf{x})_{L_{0}}$ are equivalent, to within a possible phase factor, by the spherical tensor coupled commutator relations (F6) and (F7), except in the scalar case $L_{0}=0$, where they differ by a nonzero constant $\left(c\right.$-number) commutator: $(\mathbf{x} \times \mathbf{k})_{L_{0}}=(-)^{L_{0}}(\mathbf{k} \times \mathbf{x})_{L_{0}}-$ $\sqrt{3} i \delta_{L_{0}, 0}$.

The coupling with $L_{0}=1$, in its isoscalar form, yields the orbital angular momentum operator $\mathbf{L}$. That is, the total orbital angular momentum of the nucleons, relative to the origin, is represented by the operator

$$
\mathbf{L}=\sum_{i} \mathbf{x}_{i} \times \mathbf{k}_{i}=U\left[(-i \sqrt{2})(\mathbf{x} \times \mathbf{k})_{1}\right],
$$

which we may equivalently denote in spherical tensor form as the rank-1 spherical tensor $L_{1}$. Here we make use of the relation $\boldsymbol{\eta} \times \boldsymbol{\xi}=-i \sqrt{2}(\boldsymbol{\eta} \times \boldsymbol{\xi})_{1}$ between the spherical tensor product of rank 1 and the standard vector cross product. Note that we consider here the dimensionless angular momentum operator, with dimensionless eigenvalues $L(L+1)$, commonly encountered in the nuclear many-body literature, in terms which the usual physical angular momentum operator may be recovered as $\mathbf{L}^{\text {phys }}=\hbar \mathbf{L}$. We thus obtain the identifications $\kappa \rightarrow-i \sqrt{2}, \boldsymbol{\eta} \rightarrow \mathbf{x}, \boldsymbol{\xi} \rightarrow \mathbf{k}$, and $L_{0} \rightarrow 1$, as given in Table I.

The isoscalar and isovector forms may be combined as usual, by (52), to extract the proton and neutron orbital angular momentum operators separately. These enter, along with the proton and neutron spin operators, into the leading-order or impulse-approximation $M 1$ transition operator as [104, 144, 149]

$$
\mathbf{M}_{1}=\sqrt{\frac{3}{4 \pi}} \mu_{N}\left(g_{\ell, p} \mathbf{L}_{p}+g_{\ell, n} \mathbf{L}_{n}+g_{s, p} \mathbf{S}_{p}+g_{s, n} \mathbf{S}_{n}\right),
$$

where $g_{\ell, p}=1, g_{\ell, n}=0, g_{s, p} \approx 5.586$, and $g_{s, n} \approx-3.826$.

As usual, the appropriate operator for evaluating the physical $M 1$ transition matrix element is the corresponding intrinsic operator $\mathbf{M}_{1}^{\prime}[104$, which is defined in terms of the twobody intrinsic angular momenta $\mathbf{L}_{p}^{\prime}$ and $\mathbf{L}_{n}^{\prime}$. Two-body matrix elements of these operators for use in a many-body calculation may thus be evaluated either from relative two-body matrix elements or by the separable approach, again using generic results for bilinear operators (Sec. V).

The one-body spin operators $\mathbf{S}_{p}$ and $\mathbf{S}_{n}$ are already Galilean-invariant, so their contributions to the $\mathbf{M}_{1}$ operator are already those found in the c.m. frame. However, for computational convenience, these operators may also be reexpressed as $A$-dependent twobody operators (the requisite expressions are provided for reference in Appendix D), so that $\mathbf{M}_{1}^{\prime}$ may be represented as a pure two-body operator and thus entirely expressed in terms of two-body matrix elements. 
When the wave function is known to factorize with pure harmonic oscillator $0 s$ motion for the center of mass, the naive one-body form of the $M 1$ operator given in (A18) may be used. In this case, the matrix elements of the center-of-mass contributions vanish by angular momentum selection rules, by the analysis of Sec. $\mathrm{VB}$, much as noted for the quadrupole operator above (Sec. A 1).

\section{Bilinears in harmonic oscillator ladder operators}

The bilinear couplings $\left(\mathbf{c}^{\dagger} \times \mathbf{c}\right)_{L_{0}}$ or $\left(\mathbf{c} \times \mathbf{c}^{\dagger}\right)_{L_{0}}$ of an oscillator creation and annihilation ${ }^{10}$ operator are possible for $L_{0}=0,1,2$, while $\left(\mathbf{c}^{\dagger} \times \mathbf{c}^{\dagger}\right)_{L_{0}}$ and $(\mathbf{c} \times \mathbf{c})_{L_{0}}$ are only nonvanishing for $L_{0}=0,2$. The expressions $\left(\mathbf{c}^{\dagger} \times \mathbf{c}\right)_{L_{0}}$ and $\left(\mathbf{c} \times \mathbf{c}^{\dagger}\right)_{L_{0}}$ are equivalent, to within a phase factor, by the spherical tensor coupled commutator relations ( $\overline{\mathrm{F} 6}$ ) and (F7), except in the scalar case $L_{0}=0$, where they differ by a $c$-number commutator: $\left(\mathbf{c} \times \mathbf{c}^{\dagger}\right)_{L_{0}}=(-)^{L_{0}}\left(\mathbf{c}^{\dagger} \times \mathbf{c}\right)_{L_{0}}-\sqrt{3} \delta_{L_{0}, 0}$. The definitions and properties of the single-particle, relative, and intrinsic forms of the harmonic oscillator ladder operators are reviewed in Appendix F. Bilinears in the coordinates and/or momenta may, of course, be reexpressed in terms of bilinears in oscillator ladder operators via the relations (F5), giving, e.g.,

$$
b^{-2} Q_{2}=\sqrt{\frac{15}{8 \pi}}\left[\left(\mathbf{c}^{\dagger} \times \mathbf{c}\right)_{2}+\frac{1}{2}\left(\mathbf{c}^{\dagger} \times \mathbf{c}^{\dagger}\right)_{2}+\frac{1}{2}(\mathbf{c} \times \mathbf{c})_{2}\right]
$$

for the mass quadrupole operator of Sec. A 1 .

Generators for the U(3) group of a three-dimensional harmonic oscillator are given in Cartesian form by $C_{r s}=\frac{1}{2}\left(c_{r}^{\dagger} c_{s}+c_{s} c_{r}^{\dagger}\right)=c_{r}^{\dagger} c_{s}+\frac{1}{2} \delta_{r s}(r, s=x, y, z)$. Elliot's realization of $\mathrm{U}(3)$ for the nuclear problem [98, 99] makes use of the physical subgroup chain $\mathrm{U}(3)=$ $\mathrm{U}(1) \times[\mathrm{SU}(3) \supset \mathrm{SO}(3)]$, which incorporates the $\mathrm{SO}(3)$ orbital angular momentum group. The set of 9 Cartesian generators $C_{r s}$ is then more conveniently transformed to a set of spherical tensor generators,

$$
\begin{aligned}
H_{0} & =\frac{1}{2}\left(\mathbf{c}^{\dagger} \cdot \mathbf{c}+\mathbf{c} \cdot \mathbf{c}^{\dagger}\right)=N+\frac{3}{2} \\
L_{1} & =-\sqrt{2}\left(\mathbf{c}^{\dagger} \times \mathbf{c}\right)_{1} \\
\mathcal{Q}_{2} & =\sqrt{6}\left(\mathbf{c}^{\dagger} \times \mathbf{c}\right)_{2},
\end{aligned}
$$

again with a total of nine components. Here the (dimensionless) harmonic oscillator Hamiltonian $H_{0}$ is the generator of the trivial Abelian U(1) group, $L_{1}$ is the familiar orbital angular momentum operator of (A17), now written in terms of ladder operators, and $\mathcal{Q}_{2}$ is the $\mathrm{SU}(3)$ quadrupole tensor. These operators close under commutation, with spherical tensor coupled commutators $\left[L_{1}, L_{1}\right]_{1}=-\sqrt{2} L_{1},\left[\mathcal{Q}_{2}, L_{1}\right]_{2}=-\sqrt{6} \mathcal{Q}_{2}$, and $\left[\mathcal{Q}_{2}, \mathcal{Q}_{2}\right]_{1}=3 \sqrt{10} L_{1}$, and all other commutators vanishing [99]. The resulting bilinear operators of the type $\left(\mathbf{c}^{\dagger} \times \mathbf{c}\right)_{L_{0}}$

${ }^{10}$ In these spherical tensor coupled products, the symbol $\mathbf{c}$ represents the rank- 1 covariant spherical tensor operator $c_{1}$ with components obtained from the Cartesian vector c (e.g., Sec. 5.8 of Ref. [150]) by A3]. Care must be taken in comparison with the literature, where the spherical tensor $\tilde{c}_{1}$, defined as the covariant adjoint of $c_{1}^{\dagger}$, is commonly used, yielding expressions of the form, e.g., $\left(c_{1}^{\dagger} \times \tilde{c}_{1}\right)_{L_{0}}$. We may have either $\tilde{c}_{1}= \pm c_{1}$, depending upon the convention adopted for the covariant adjoint in a given reference, as detailed in Sec. F1. 
are summarized in Table $\mathrm{I}$. For the $A$-body system, the Elliott $\mathrm{U}(3)$ generators are realized as the corresponding one-body operators, that is, summed over nucleons.

In particular, for $L_{0}=0$, the $A$-particle harmonic oscillator Hamiltonian,

$$
H_{0}=\sum_{i} \frac{1}{2}\left(\mathbf{c}_{i}^{\dagger} \cdot \mathbf{c}_{i}+\mathbf{c}_{i} \cdot \mathbf{c}_{i}^{\dagger}\right)=N+\frac{3}{2} A,
$$

is related to the $A$-particle harmonic oscillator number operator $N$ by a $c$-number offset $\frac{3}{2} A$, representing the aggregate zero-point energies of the $A$ particles. The number operator is simply

$$
N=\sum_{i} \mathbf{c}_{i}^{\dagger} \cdot \mathbf{c}_{i}=U\left[(-\sqrt{3})\left(\mathbf{c}^{\dagger} \times \mathbf{c}\right)_{0}\right] .
$$

We thus obtain the identifications $\kappa \rightarrow-\sqrt{3}, \boldsymbol{\eta} \rightarrow \mathbf{c}^{\dagger}, \boldsymbol{\xi} \rightarrow \mathbf{c}$, and $L_{0} \rightarrow 0$, as given in Table I.

For $L_{0}=2$, the $\mathrm{SU}(3)$ quadrupole operator may be reexpressed in terms of the coordinate and momentum bilinears of Secs. A1 A 2, which are then scaled by appropriate powers of the oscillator length $b$ from (F2) to produce a dimensionless result, as

$$
\mathcal{Q}_{2}=\sum_{i} \sqrt{\frac{3}{2}}\left[b^{-2}\left(\mathbf{x}_{i} \times \mathbf{x}_{i}\right)_{2}+b^{2}\left(\mathbf{k}_{i} \times \mathbf{k}_{i}\right)_{2}\right],
$$

and thus 99

$$
\mathcal{Q}_{2}=\sum_{i} \sqrt{\frac{4 \pi}{5}}\left[b^{-2} x_{i}^{2} Y_{2}\left(\hat{\mathbf{x}}_{i}\right)+b^{2} k_{i}^{2} Y_{2}\left(\hat{\mathbf{k}}_{i}\right)\right] .
$$

The SU(3) quadrupole tensor may thus be recognized as a linear combination of the mass quadrupole operator of (A10) and its momentum-space analog.

An important consequence of the bilinear forms of these $\mathrm{U}(3)$ generators is that, by (42), each generator separates into intrinsic and center-of-mass parts [112]. That is, rearranging (42) to isolate the one-body operator on the left-hand side, we have

$$
\underbrace{\sum_{i}\left(\boldsymbol{\eta}_{i} \times \boldsymbol{\xi}_{i}\right)_{L_{0}}}_{T_{L_{0}}}=\underbrace{\sum_{i}\left(\boldsymbol{\eta}_{i}^{\prime} \times \boldsymbol{\xi}_{i}^{\prime}\right)_{L_{0}}}_{T_{L_{0}}^{\prime}}+\underbrace{\frac{1}{A}\left[\left(\sum_{i} \boldsymbol{\eta}_{i}\right) \times\left(\sum_{j} \boldsymbol{\xi}_{j}\right)\right]_{L_{0}}}_{T_{\text {c.m. }, L_{0}}},
$$

yielding a decomposition of a general bilinear operator $T_{L_{0}}$ into intrinsic and c.m. parts as $T_{L_{0}}=T_{L_{0}}^{\prime}+T_{\text {c.m., } L_{0}}$. Thus, in particular, $H_{0}=H_{0}^{\prime}+H_{0, \text { c.m. }}$ (and, similarly, $N=N^{\prime}+N_{\text {c.m. }}$ ), $\mathbf{L}=\mathbf{L}^{\prime}+\mathbf{L}_{\text {c.m. }}$, and $\mathcal{Q}_{2}=\mathcal{Q}_{2}^{\prime}+\mathcal{Q}_{\text {c.m. } .2}$. When the separation is made into intrinsic and centerof-mass parts of the $\mathrm{U}(1)$ generator $H_{0}$, then $H_{0, \text { c.m. }}=N_{\text {c.m. }}+3 / 2$ for the center-of-mass oscillator, while the remaining $3(A-1) / 2$ units of zero-point energy reside in the intrinsic Hamiltonian, which is related to the intrinsic number operator by $H_{0}^{\prime}=N^{\prime}+3(A-1) / 2$.

The U(3) symmetry group of the harmonic oscillator lies within the larger $\mathrm{Sp}(3, \mathbb{R})$ dynamical group of the oscillator, which provides raising and lowering operators connecting different oscillator shells (e.g., Ref. [127]). In Cartesian form, the generators of $\operatorname{Sp}(3, \mathbb{R})$ consist of the symplectic raising operators $A_{r s}=A_{s r}=c_{r}^{\dagger} c_{s}^{\dagger}$, which carry +2 oscillator quanta, and the symplectic lowering operators $B_{r s}=B_{s r}=c_{r} c_{s}$, which carry -2 oscillator quanta, along with the number conserving $\mathrm{U}(3)$ generators $C_{r s}$ defined above, giving a total of twenty-one components. 
To make use of the full machinery of tensor operators in a symmetry adapted many-body basis, it is more useful to transform these generators to $\mathrm{SU}(3) \supset \mathrm{SO}(3)$-coupled form. This is accomplished making use of the property that the oscillator creation and annihilation operators form $(1,0)$ and $(0,1) \mathrm{SU}(3)$ tensors, respectively. Written as $\mathrm{SU}(3)$ coupled products, the generators are then [151, 152$]^{11}$

$$
\begin{array}{cc}
A_{L}^{(2,0)}=\frac{1}{\sqrt{2}}\left(c^{\dagger(1,0)} \times c^{\dagger(1,0)}\right)_{L}^{(2,0)} & L=0,2 \\
B_{L}^{(0,2)}=\frac{1}{\sqrt{2}}\left(c^{(0,1)} \times c^{(0,1)}\right)_{L}^{(0,2)} & L=0,2 \\
C_{L}^{(1,1)}=\sqrt{2}\left(c^{\dagger(1,0)} \times c^{(0,1)}\right)_{L}^{(1,1)} & L=1,2 \\
H_{0}^{(0,0)}=\frac{\sqrt{3}}{2}\left[\left(c^{\dagger(1,0)} \times c^{(0,1)}\right)_{0}^{(0,0)}+\left(c^{(0,1)} \times c^{\dagger(1,0)}\right)_{0}^{(0,0)}\right] .
\end{array}
$$

Using $\mathrm{SU}(3) \supset \mathrm{SO}(3)$ reduced coupling coefficients under standard phase conventions [153] to explicitly evaluate the $\mathrm{SU}(3)$ coupled products in A26) gives

$$
\begin{array}{rlrl}
A_{0}^{(2,0)}=-\frac{1}{\sqrt{2}}\left(\mathbf{c}^{\dagger} \times \mathbf{c}^{\dagger}\right)_{0} & A_{2}^{(2,0)}=+\frac{1}{\sqrt{2}}\left(\mathbf{c}^{\dagger} \times \mathbf{c}^{\dagger}\right)_{2} \\
B_{0}^{(0,2)}=-\frac{1}{\sqrt{2}}(\mathbf{c} \times \mathbf{c})_{0} & B_{2}^{(0,2)}=+\frac{1}{\sqrt{2}}(\mathbf{c} \times \mathbf{c})_{2} \\
C_{1}^{(1,1)}=-\sqrt{2}\left(\mathbf{c}^{\dagger} \times \mathbf{c}\right)_{1} & C_{2}^{(1,1)}=+\sqrt{2}\left(\mathbf{c}^{\dagger} \times \mathbf{c}\right)_{2} \\
H_{0}^{(0,0)}=-\frac{\sqrt{3}}{2}\left[\left(\mathbf{c}^{\dagger} \times \mathbf{c}\right)_{0}+\left(\mathbf{c} \times \mathbf{c}^{\dagger}\right)_{0}\right],
\end{array}
$$

from which we recognize $C_{1}^{(1,1)}=L_{1}, \sqrt{3} C_{2}^{(1,1)}=\mathcal{Q}_{2}$, and $H_{0}^{(0,0)}=H_{0}=N+3 / 2$. The correspondence to the generic bilinear operator is summarized in Table [1. Again, as for the $\mathrm{U}(3)$ generators above, the generators acting on an $A$-body system are obtained as the resulting one-body operators, summed over nucleons. The intrinsic forms of the $\operatorname{Sp}(3, \mathbb{R})$ generators given in (38) of Ref. [96] or (2) of Ref. [154] may be recognized as the intermediate step of our decomposition found in (41).

\section{Appendix B: Proton-neutron mass difference and corrections to kinetic energy}

Throughout this work, we have assumed that the proton and neutron masses could be approximated as a single "nucleon mass" $m_{N}$ (see footnote1). It is well known that including the proton-neutron mass difference induces an isovector correction to the kinetic energy [155]. Here we derive that correction more completely and extend the derivation to the intrinsic kinetic energy.

Let us define the mean nucleon mass and the nucleon mass deviation by

$$
m_{N}=\frac{1}{2}\left(m_{p}+m_{N}\right) \quad \Delta_{m}=\frac{1}{2}\left(m_{p}-m_{n}\right) .
$$

${ }^{11}$ Here $T_{L}^{(\lambda, \mu)}$ represents the spherical tensor component with $\mathrm{SO}(3)$ angular momentum $L$ of an $\mathrm{SU}(3)$ tensor forming an irrep with Elliott labels $(\lambda, \mu)[127$. 
We can then define the relative mass deviation as

$$
\delta_{m}=\frac{\Delta_{m}}{m_{N}}=\frac{m_{p}-m_{n}}{m_{p}+m_{n}},
$$

and write the mass of the $i$ th nucleon as

$$
m_{i}=m_{N}\left(1+\tau_{i 0} \delta_{m}\right) .
$$

The total nuclear mass is then

$$
M=\left[A+(Z-N) \delta_{m}\right] m_{N} .
$$

We now examine the total kinetic energy of a system of $A$ nucleons, defined by

$$
T=U\left[\frac{\hbar^{2} k^{2}}{2 m}\right]=\sum_{i} \frac{\hbar^{2} k_{i}^{2}}{2 m_{i}} .
$$

Expressing the nucleon-dependence of the mass in terms of $\tau_{i 0}$ via $(\overline{\mathrm{B} 3})$, this becomes

$$
T=\sum_{i} \frac{\hbar^{2} k_{i}^{2}}{2 m_{N}\left(1+\tau_{i 0} \delta_{m}\right)} .
$$

Expanding the denominator of the summand as a geometric series, and resumming the series ${ }^{12}$ removes $\tau_{i 0}$-dependence from the denominator, giving

$$
T=\frac{1}{1-\delta_{m}^{2}} \underbrace{\sum_{i} \frac{\hbar^{2} k_{i}^{2}}{2 m_{N}}}_{T_{\mathrm{IS}}}-\frac{\delta_{m}}{1-\delta_{m}^{2}} \underbrace{\sum_{i} \frac{\hbar^{2} k_{i}^{2} \tau_{i 0}}{2 m_{N}}}_{T_{\mathrm{IV}}} .
$$

We thus obtain an expression for the kinetic energy which separates into isoscalar and isovector terms. The prefactor $\left(1-\delta_{m}^{2}\right)^{-1}$ is second-order in the nucleon mass difference and is thus neglected in (2.18) of Ref. [155].

Now we turn to the intrinsic kinetic energy $T^{\prime}$, obtained by the substitution $\mathbf{k}_{i} \rightarrow \mathbf{k}_{i}^{\prime}$. However, recall that the form of the intrinsic coordinates and momenta which we have thus far been using in such substitutions, given in (18), is obtained by neglecting the protonneutron mass difference. We must instead return to the fundamental definition, given in (14), which may be reexpressed in terms of the masses $m_{i}$ as

$$
\mathbf{x}_{i}^{\prime}=\mathbf{x}_{i}-\frac{1}{M} \sum_{j} m_{j} \mathbf{x}_{j} \quad \mathbf{k}_{i}^{\prime}=\mathbf{k}_{i}-\frac{m_{i}}{M} \sum_{j} \mathbf{k}_{j} .
$$

Thus, we obtain an intrinsic kinetic energy

$$
T^{\prime}=\sum_{i} \frac{\hbar^{2}}{2 m_{i}}\left(\mathbf{k}_{i}-\frac{m_{i}}{M} \sum_{j} \mathbf{k}_{j}\right) \cdot\left(\mathbf{k}_{i}-\frac{m_{i}}{M} \sum_{k} \mathbf{k}_{k}\right) .
$$

12 Namely, recognizing that $\left(\tau_{i 0}\right)^{2}=1$, we obtain $\left(1+\tau_{i 0} \delta_{m}\right)^{-1}=\sum_{k=0}^{\infty}\left(-\tau_{i 0}\right)^{k} \delta_{m}^{k}=\sum_{\ell=0}^{\infty}\left(\left(-\tau_{i 0}\right)^{2 \ell} \delta_{m}^{2 \ell}+\right.$ $\left.\left(-\tau_{i 0}\right)^{2 \ell+1} \delta_{m}^{2 \ell+1}\right)=\sum_{\ell=0}^{\infty}\left(1-\tau_{i 0} \delta_{m}\right)\left(\delta_{m}^{2}\right)^{\ell}=\left(1-\tau_{i 0} \delta_{m}\right) /\left(1-\delta_{m}^{2}\right)$. 
Expanding, and using the identity $\sum_{i} m_{i}=M$ and to recognize and combine like terms, we arrive at

$$
T^{\prime}=\sum_{i} \frac{\hbar^{2} k_{i}^{2}}{2 m_{i}}-\frac{\hbar^{2}}{2 M}\left(\sum_{i} \mathbf{k}_{i}\right) \cdot\left(\sum_{j} \mathbf{k}_{j}\right) .
$$

This expression is recognizable as a decomposition of the intrinsic operator as the total (one-body) operator less a center-of-mass contribution, generalizing (2) to unequal nucleon masses.

Then, to obtain a separation into one-body and separable two-body parts, we multiply out the product of sums appearing in the second term of (B10) and extract the diagonal terms from the resulting double sum, while eliminating the explicit nucleon mass dependence from the denominator of the first term as in $(\mathrm{B} 7)$, to obtain

$$
\begin{aligned}
T^{\prime}=\left(\frac{1}{1-\delta_{m}^{2}}-\frac{1}{A+(Z-N) \delta_{m}}\right) & \underbrace{\sum_{i}^{\frac{\hbar^{2} k_{i}^{2}}{2 m_{N}}}}_{T_{\mathrm{IS}}} \\
& -\frac{\delta_{m}}{1-\delta_{m}^{2}} \underbrace{\sum_{i} \frac{\hbar^{2} k_{i}^{2} \tau_{i 0}}{2 m_{N}}}_{T_{\mathrm{IV}}}-\frac{1}{A+(Z-N) \delta_{m}} \sum_{i j}^{\prime} \frac{\hbar^{2} \mathbf{k}_{i} \cdot \mathbf{k}_{j}}{2 m_{N}},
\end{aligned}
$$

thereby generalizing (4) to unequal nucleon masses. In the one-body part, we recognize a separation into isoscalar and isovector terms, involving the same operators $T_{\mathrm{IS}}$ and $T_{\mathrm{IV}}$, respectively, as in $(\bar{B} 7)$. The two-body part is isoscalar and simply reflects the nucleon mass difference through its $(Z-N)$-dependent prefactor. However, if the nucleon mass were instead taken as $m_{N}=\left(Z m_{p}+N m_{n}\right) / A$ (see footnote 1), note that the prefactor of the two-body term would then simply be $1 / A$, and the two-body contribution would reduce to that in (B7).

\section{Appendix C: Summation identities for two-body operators}

A couple of straightforward summation identities are essential in working with one-body and two-body operators.

In particular, any one-body operator may be "upgraded" to a two-body operator. Observe that

$$
\sum_{i j}^{\prime}\left(u_{i}+u_{j}\right)=2(A-1) \sum_{i} u_{i},
$$

and thus we recognize that

$$
V\left[u_{1}+u_{2}\right]=(A-1) U[u] .
$$

In the definition (8) of the two-body operator, the expression $v_{i j}$ is only taken to be defined for $i \neq j$. However, in the applications considered here, where $v_{i j}$ arises as a product operator of the form $v_{i j}=\eta_{i} \xi_{j}$, we also naturally encounter unrestricted sums of $v_{i j}$ over the two particle indices $i$ and $j$. Such a sum can be broken into one-body and two-body parts, as

$$
\sum_{i j} v_{i j}=\sum_{i} v_{i i}+\sum_{i j}^{\prime} v_{i j},
$$


by extracting the diagonal $(i=j)$ terms from the double sum. If $v_{i j}$ is known to be symmetric under interchange of the particle indices $(i \leftrightarrow j)$, the expression on the right hand side can immediately be recognized as $U[u]+2 V[v]$, where $u$ is defined on the single-particle space as $u=v_{11}$, and $v$ is defined as usual on the two-particle space as $v=v_{12}=v_{21}$. More generally, if $v_{i j}$ cannot be assumed to be symmetric, we must symmetrize the summand of the double sum, to obtain

$$
\sum_{i j} v_{i j}=\sum_{i} v_{i i}+\frac{1}{2} \sum_{i j}^{\prime}\left(v_{i j}+v_{j i}\right),
$$

and recognize the sums on the right hand side as representing one-body and two-body operators, respectively, to obtain

$$
\sum_{i j} v_{i j}=U\left[v_{11}\right]+V\left[v_{12}+v_{21}\right] .
$$

\section{Appendix D: Two-body realization of spin operators}

The total spin operators (proton and neutron spin or, equivalently, isoscalar and isovector spin) are inherently independent of the c.m. coordinate degree of freedom. They are thus already intrinsic operators. Nonetheless, they are taken in linear combination with the orbital angular momentum operators to generate the $M 1$ operator, and, while the intrinsic orbital angular momentum operators are two-body operators, these spin operators are only one-body operators. To incorporate them into the evaluation of two-body matrix elements for the $M 1$ operator, it is convenient to use the relation (C2) to upgrade them to two-body operators.

Thus, we note that the one-body total spin operator

$$
\mathbf{S}=U[\mathbf{s}]=\sum_{i} \mathbf{s}_{i}
$$

may be rewritten, using identity (C1), as an $A$-dependent two-body operator, as

$$
\mathbf{S}=\frac{1}{A-1} \frac{1}{2} \sum_{i j}^{\prime}\left(\mathbf{s}_{i}+\mathbf{s}_{j}\right)=\frac{1}{A-1} V\left[\mathbf{S}_{\mathrm{rel}}\right],
$$

where $\mathbf{S}_{\text {rel }}=\mathbf{s}_{1}+\mathbf{s}_{2}$ is the total spin operator on the two-body system.

Then, the one-body proton and neutron spin operators are

$$
\mathbf{S}_{\alpha}=U\left[\mathbf{s} \delta_{\alpha}\right]=\sum_{i} \mathbf{s}_{i} \delta_{i, \alpha} .
$$

These operators separate into manifestly isoscalar and isovector parts, much as for the proton or neutron dipole operator in (24). Inserting the definition (23) for $\delta_{\alpha}$ gives

$$
\mathbf{S}_{\alpha}=\frac{1}{2} \underbrace{\left[\sum_{i} \mathbf{s}_{i}\right]}_{\mathbf{S}}+\frac{1}{2} \alpha \underbrace{\left[\sum_{i} \mathbf{s}_{i} \tau_{i 0}\right]}_{\mathbf{S}_{\mathrm{IV}}},
$$


that is, $\mathbf{S}_{\alpha}=\frac{1}{2} \mathbf{S}+\frac{1}{2} \alpha \mathbf{S}_{\mathrm{IV}}$, where $\mathbf{S}$ is simply the isoscalar total spin operator from (D1), while

$$
\mathbf{S}_{\mathrm{IV}}=U\left[\mathbf{s} \tau_{0}\right]=\sum_{i} \mathbf{s}_{i} \tau_{i 0}
$$

is the isovector spin operator. Again using identity (C1), the isovector spin operator may be rewritten as an $A$-dependent two-body operator, as

$$
\mathbf{S}_{\mathrm{IV}}=\frac{1}{A-1} \frac{1}{2} \sum_{i j}^{\prime}\left(\mathbf{s}_{i} \tau_{i 0}+\mathbf{s}_{j} \tau_{j 0}\right)=\frac{1}{A-1} V\left[\mathbf{S}_{\mathrm{IV}, \mathrm{rel}}\right]
$$

where $\mathbf{S}_{\mathrm{IV}, \mathrm{rel}}=\mathbf{s}_{1} \tau_{01}+\mathbf{s}_{2} \tau_{02}$ is the isovector spin operator on the two-body system.

\section{Appendix E: Isospin-reduced matrix elements: Dipole and bilinear operators}

Since the spatial and isospin dependences of the isovector parts of the relative dipole operator (Sec. IV) and relative intrinsic bilinear operator (Sec. VP) factorize, it is straightforward to evaluate the isospin contribution to two-body matrix elements. Let us write the two-body states as $|\gamma T\rangle$, with isospin $T=0,1$, where $\gamma$ represents all quantum numbers other than isospin (typically, for an $L S$-coupled relative oscillator basis, $\gamma \equiv N_{\text {rel }} L S J$ ). Since we are working with basis states of good isospin and isovector operators (i.e., spherical tensors of rank 1 under isospin rotations, carrying definite isospin $\left.T_{0}=1\right)$ it is most streamlined to work with isospin-reduced matrix elements $\left\langle\gamma^{\prime} T^{\prime}\|\cdots\| \gamma T\right\rangle$.

The reduced matrix element $\left\langle\gamma^{\prime} T^{\prime}\left\|\mathbf{D}_{\mathrm{IV} \text {,rel }}\right\| \gamma T\right\rangle$, of the isovector relative dipole operator $\mathbf{D}_{\text {IV,rel }}$, factorizes into a spatial-spin part $\propto\left\langle\gamma^{\prime}\left|\mathbf{x}_{\text {rel }}\right| \gamma\right\rangle$, which depends on the particular choice of basis wave functions, and an isospin part $\left\langle T^{\prime}\left\|\boldsymbol{\tau}_{1}-\boldsymbol{\tau}_{2}\right\| T\right\rangle$, which is independent of the details of the basis. We can thus note for reference these latter matrix elements on the two-body system. Since the operator $\boldsymbol{\tau}_{1}-\boldsymbol{\tau}_{2}$ is isovector, the matrix element between $T=0$ states vanishes by the angular momentum triangularity selection rule: $\left\langle 0 \| \boldsymbol{\tau}_{1}-\right.$ $\left.\boldsymbol{\tau}_{2} \| 0\right\rangle=0$. The operator likewise has vanishing expectation value within a $T=1$ state: $\left\langle 1\left\|\boldsymbol{\tau}_{1}-\boldsymbol{\tau}_{2}\right\| 1\right\rangle=0$. (The vectorial interpretation is that this state represents the aligned coupling of the two $T=1 / 2$ isospins of the nucleons. The isospin projections of the two nucleons along any axis in isospin space must therefore align, and their difference along the axis thus cancels.) The remaining matrix element, between $T=0$ and $T=1$ states, may be evaluated by standard angular momentum coupling methods (namely, Racah's reduction formulas), giving $\left\langle 0\left\|\boldsymbol{\tau}_{1}-\boldsymbol{\tau}_{2}\right\| 1\right\rangle=-2 \sqrt{3}$. We follow the normalization and phase convention of Rose [156] for the Wigner-Eckart theorem when defining the reduced matrix element, i.e., $\left\langle J^{\prime} M^{\prime}\left|T_{J_{0} M_{0}}\right| J M\right\rangle=\left(J M J_{0} M_{0} \mid J^{\prime} M^{\prime}\right)\left\langle J^{\prime}|| T_{J_{0}}|| J\right\rangle$. To convert isospin-reduced matrix elements to the normalization convention of Edmonds [157], they may be multipled by $\left(2 T^{\prime}+1\right)^{1 / 2}$, i.e., in this case just unity.

The situation is, in fact, much simpler for the bilinear opertors. Recall, from Sec. VB, that we must evaluate two-body matrix elements of the isoscalar relative operator $T_{\text {rel }, L_{0}}$ (48) and its isovector counterpart $T_{\text {rel, } L_{0}}\left(2 T_{0}\right)$, in the two-body basis. The isospin-reduced matrix elements of these two operators are closely related:

$$
\left\langle\gamma^{\prime} T^{\prime}\left\|T_{\text {rel }, L_{0}}(2 \mathbf{T})\right\| \gamma T\right\rangle=\left\langle\gamma^{\prime}\left|T_{\text {rel }, L_{0}}\right| \gamma\right\rangle\left\langle T^{\prime}\|2 \mathbf{T}\| T\right\rangle .
$$

The isospin-reduced matrix element follows from the well-known identity for the reduced matrix element of the angular momentum operator in an angular momentum basis: $\left\langle J^{\prime}\|\mathbf{J}\| J\right\rangle=$ 
$\delta_{J^{\prime} J}[J(J+1)]^{1 / 2}$. It is nonvanishing only between the $T=1$ states, for which we have $\langle 1\|2 \mathbf{T}\| 1\rangle=2 \sqrt{2}$ in the convention of Rose. Again, to convert to the normalization convention of Edmonds [157], this matrix element may be multiplied by $\left(2 T^{\prime}+1\right)^{1 / 2}$, i.e., $\sqrt{3}$.

\section{Appendix F: Harmonic oscillator ladder operators: Single-particle, relative, and intrinsic}

\section{Ladder operators in three dimensions}

Several of the intrinsic operators considered in this work are more naturally represented in terms of oscillator creation and annihilation, or ladder, operators than directly in terms of the coordinate and momentum operators. After reviewing the basic definitions and relations, we therefore lay out properties of the ladder operators defined on the two-body relative (Sec. III B) and $A$-body intrinsic (Sec. IIIC) degrees of freedom, as well as their complementary c.m. degrees of freedom. Care must be taken with conventional factors arising in the definitions of coordinates and momenta, as in Secs. III C III B.

First, let us recall the ladder operators defined for a three-dimensional isotropic harmonic oscillator [109, 150], in terms of the coordinate vector $\mathbf{x}$ and conjugate momentum vector $\mathbf{p}=\hbar \mathbf{k}$. The oscillator problem is defined by the Hamiltonian

$$
H=\frac{\hbar^{2}}{2 m} \mathbf{k}^{2}+\frac{m \omega^{2}}{2} \mathbf{x}^{2}
$$

with mass $m$ and oscillator frequency $\omega$ as parameters. Although this expression in (F1) is perhaps the most familiar form for the Hamiltonian, it may be parametrized instead in terms of the oscillator length $b=[\hbar /(m \omega)]^{1 / 2}$, which allows the Hamiltonian to be written more symmetrically in the coordinate and momentum as

$$
H=\frac{\hbar \omega}{2}\left(b^{2} \mathbf{k}^{2}+b^{-2} \mathbf{x}^{2}\right)
$$

Thus, $\mathbf{x}$, with dimensions of length, and $\mathbf{k}$, with dimensions of inverse length, are each scaled by the appropriate power of $b$ to make the quantity in parentheses dimensionless. The oscillator length is also significant in that it determines the overall length scale, or dilation, of the eigenfunctions obtained from this oscillator Hamiltonian (F2) [147], but here we are primarily concerned not with the eigenfunctions but with the operators.

The oscillator Hamiltonian (F2) may then be reexpressed in terms of the oscillator creation operator $\mathbf{c}^{\dagger}$ and annihilation operator $\mathbf{c}$, as $H=\hbar \omega\left(\mathbf{c}^{\dagger} \cdot \mathbf{c}+3 / 2\right)$. The traditional expression for the creation operator is

$$
\mathbf{c}^{\dagger}=\left(\frac{m \omega}{2 \hbar}\right)^{1 / 2} \mathbf{x}-i\left(\frac{\hbar}{2 m \omega}\right)^{1 / 2} \mathbf{k}
$$

The lowering operator $\mathbf{c}=\left(\mathbf{c}^{\dagger}\right)^{\dagger}$ is defined by taking the adjoint of $\mathbf{c}^{\dagger}$, that is, more precisely, conjugating each Cartesian component. As with the Hamiltonian, these operators may be reparametrized in terms of the oscillator length to yield more symmetric expressions

$$
\mathbf{c}^{\dagger}=\frac{1}{\sqrt{2}}\left(b^{-1} \mathbf{x}-i b \mathbf{k}\right) \quad \mathbf{c}=\frac{1}{\sqrt{2}}\left(b^{-1} \mathbf{x}+i b \mathbf{k}\right) .
$$


Inverting for the coordinate and momentum equivalently yields

$$
b^{-1} \mathbf{x}=\frac{1}{\sqrt{2}}\left(\mathbf{c}+\mathbf{c}^{\dagger}\right) \quad b \mathbf{k}=-\frac{i}{\sqrt{2}}\left(\mathbf{c}-\mathbf{c}^{\dagger}\right) .
$$

The total number of oscillator excitations (along all Cartesian axes) is then counted by the oscillator number operator $N=\mathbf{c}^{\dagger} \cdot \mathbf{c}=\sum_{r} c_{r}^{\dagger} c_{r}(r=1,2,3)$, in terms of which the Hamiltonian operator in $(\mathrm{F} 2)$ is simply $H=\hbar \omega(N+3 / 2)$.

Recall the canonical commutation properties, for the Cartesian components, which will be useful in understanding the relations of intrinsic, relative, and c.m. oscillator ladder operators below. Since the coordinates and momenta obey canonical commutation relations $\left[x_{r}, k_{s}\right]=\delta_{r s} i\left(\right.$ with $\left.\left[x_{r}, x_{s}\right]=\left[k_{r}, k_{s}\right]=0\right)$, it follows by (F4) that the Cartesian components of $\mathbf{c}$ and $\mathbf{c}^{\dagger}$ obey canonical commutation relations $\left[c_{r}, c_{s}^{\dagger}\right]=\delta_{r s}$ (with $\left.\left[c_{r}, c_{s}\right]=\left[c_{r}^{\dagger}, c_{s}^{\dagger}\right]=0\right)$.

The canonical commutators for vector operators can be more concisely expressed if we consider the spherical tensor coupled commutator [141, 158, 159]. For two spherical tensors of angular momentum (or rank) $a$ and $b$, respectively, the coupled commutator of rank $c$ is the spherical tensor defined by

$$
\left[A_{a}, B_{b}\right]_{c}=\left(A_{a} \times B_{b}\right)_{c}-(-)^{c-a-b}\left(B_{b} \times A_{a}\right)_{c}
$$

(see, e.g., Appendix of Ref. [160] for a review). From the above commutators for the Cartesian components of $\mathbf{x}$ and $\mathbf{k}$, and similarly of $\mathbf{c}$ and $\mathbf{c}^{\dagger}$, we obtain coupled commutators

$$
[\mathbf{x}, \mathbf{k}]_{L_{0}}=-\sqrt{3} i \delta_{L_{0}, 0} \quad\left[\mathbf{c}, \mathbf{c}^{\dagger}\right]_{L_{0}}=-\sqrt{3} \delta_{L_{0}, 0}
$$

while $[\mathbf{x}, \mathbf{x}]_{L_{0}}=[\mathbf{k}, \mathbf{k}]_{L_{0}}=0$ and $[\mathbf{c}, \mathbf{c}]_{L_{0}}=\left[\mathbf{c}^{\dagger}, \mathbf{c}^{\dagger}\right]_{L_{0}}=0$.

Here we are representing the annihilation operator as a spherical tensor by taking the rank-1 covariant spherical tensor $c_{1}$ obtained directly from the Cartesian vector $\mathbf{c}$ via (A3). However, care must be taken when comparing with the literature. It is also common to start from $\mathbf{c}^{\dagger}$, take the corresponding covariant spherical tensor $c_{1}^{\dagger}$, and then obtain the covariant adjoint tensor $\widetilde{\left(c_{1}^{\dagger}\right)^{\dagger}}$ (see, e.g., Sec. 4.8 of Ref. [110] or Sec. A.3 of Ref. [150] for the covariant adjoint of a spherical tensor). The resulting tensor is commonly denoted by

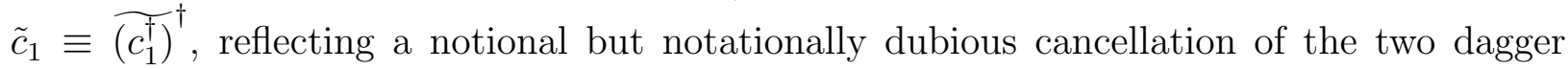
symbols (despite their substantially different definitions).

The relation between $\mathbf{c}$ (or, rather, the corresponding covariant spherical tensor $c_{1}$ ) and $\tilde{c}_{1}$ depends upon the convention in use for the covariant adjoint. If the covariant adjoint of a tensor $T_{J}$ is defined as (e.g., Refs. [150, 161])

$$
\tilde{T}_{J M}^{\dagger}=(-)^{J-M} T_{J,-M}^{\dagger}
$$

or as $\tilde{T}_{J M}^{\dagger}=(-)^{J+M} T_{J,-M}^{\dagger}$ (e.g., Ref. [147]), then we have $\tilde{c}_{1}=-c_{1}$. However, for spherical tensors of integer rank $L$, it is also common (see Sec. A.6 of Ref. [150]) to take the alternative definition, modeled on the conjugation property of the spherical harmonics,

$$
\tilde{T}_{L M}^{\dagger}=(-)^{M} T_{L,-M}^{\dagger} .
$$

In this case, we have $\tilde{c}_{1}=+c_{1}$.

In particular, the expression $\left[\tilde{A}_{a}, B_{b}^{\dagger}\right]_{c}=\hat{a} \delta_{A B} \delta_{c, 0}$ for the generic canonical commutator for bosonic ladder operators, as found in (10) of Ref. [159] or (A.8) of Ref. [160], gives 
$\left[\tilde{c}_{1}, c_{1}^{\dagger}\right]_{L_{0}}=+\sqrt{3} \delta_{L_{0}, 0}$. This may be reconciled with the opposite sign appearing on the right hand side of the expression for $\left[\mathbf{c}, \mathbf{c}^{\dagger}\right]_{L_{0}}$ given above in $(\mathrm{F} 7)$ by noting that the result of Refs. [159, 160] is derived in terms of a covariant adjoint defined under a phase convention in which $c_{1}=-\tilde{c}_{1}$. On the other hand, the sign of the $\mathrm{SU}(3)$ generator expression in (A26) is consistent with (3) of Ref. [152], as this is obtained under the phase convention in which $c_{1}=+\tilde{c}_{1}$.

\section{Relative ladder operators (two-body system)}

For the two-particle system (Sec. III B), relative and c.m. ladder operators are obtained by a unitary change of basis on the bosonic creation operators, from the single-particle ladder operators to the difference and sum operators, as

$$
\mathbf{c}_{\mathrm{rel}}^{\dagger}=\frac{1}{\sqrt{2}}\left(\mathbf{c}_{1}^{\dagger}-\mathbf{c}_{2}^{\dagger}\right) \quad \mathbf{c}_{\mathrm{c} . \mathrm{m} .}^{\dagger}=\frac{1}{\sqrt{2}}\left(\mathbf{c}_{1}^{\dagger}+\mathbf{c}_{2}^{\dagger}\right) .
$$

Such a unitary change of basis automatically preserves the canonical commutation relations (e.g., Ref. [162]). That is, starting from the canonical commutators for the individual particles, $\left[\mathbf{c}_{i}, \mathbf{c}_{j}^{\dagger}\right]_{L_{0}}=-\sqrt{3} \delta_{i j} \delta_{L_{0}, 0}(i, j=1,2)$, etc., we have $\left[\mathbf{c}_{\mathrm{rel}}, \mathbf{c}_{\mathrm{rel}}^{\dagger}\right]_{L_{0}}=\left[\mathbf{c}_{\mathrm{c} . \mathrm{m} .}, \mathbf{c}_{\mathrm{c} . \mathrm{m} .}^{\dagger}\right]_{L_{0}}=$ $-\sqrt{3} \delta_{L_{0}, 0}$ and $\left[\mathbf{c}_{\mathrm{rel}}, \mathbf{c}_{\mathrm{c} . \mathrm{m} .}^{\dagger}\right]_{L_{0}}=0$, etc.

The form of the total number operator is also preserved under a unitary change of basis

on the bosonic opeartors, that is, $N=\mathbf{c}_{1}^{\dagger} \cdot \mathbf{c}_{1}+\mathbf{c}_{2}^{\dagger} \cdot \mathbf{c}_{2}=\mathbf{c}_{\mathrm{rel}}^{\dagger} \cdot \mathbf{c}_{\mathrm{rel}}+\mathbf{c}_{\mathrm{c} . \mathrm{m} .}^{\dagger} \cdot \mathbf{c}_{\mathrm{c} . \mathrm{m} .}$. The number operator may thus be decomposed into mutually-commuting relative and c.m. contributions as $N=N_{\text {rel }}+N_{\text {c.m. }}$, with $N_{\text {rel }}=\mathbf{c}_{\text {rel }}^{\dagger} \cdot \mathbf{c}_{\text {rel }}$ and $N_{\text {c.m. }}=\mathbf{c}_{\text {c.m. }}^{\dagger} \cdot \mathbf{c}_{\text {c.m. }} \cdot$

However, care must be taken in identifying the relevant oscillator lengths, under the conventions chosen for the relative and c.m. coordinates. (Failing to properly do so, when numerically evaluating integrals for relative two-body matrix elements, for instance, leads to use of an erroneous value for the oscillator length parameter in the relative harmonic oscillator basis functions, and thus erroneous values for the matrix elements.) We recognize

$$
\begin{array}{cc}
\mathbf{c}_{\text {rel }}^{\dagger}=\frac{1}{\sqrt{2}}\left(b_{\text {rel }}^{-1} \mathbf{x}_{\text {rel }}-i b_{\text {rel }} \mathbf{k}_{\text {rel }}\right) & b_{\text {rel }}=\left\{\begin{array}{c}
\sqrt{2} \\
1
\end{array}\right\} b \\
\mathbf{c}_{\text {c.m. }}^{\dagger}=\frac{1}{\sqrt{2}}\left(b_{\text {c.m. }}{ }^{-1} \mathbf{x}_{\text {c.m. }}-i b_{\text {c.m. }} \mathbf{k}_{\text {c.m. }}\right) & b_{\text {c.m. }}=\left\{\begin{array}{c}
\frac{1}{\sqrt{2}} \\
1
\end{array}\right\} b,
\end{array}
$$

with the upper coefficient (in braces) applying under the mechanics convention and the lower coefficient applying under the symmetric convention for the relative-c.m. coordinates, as in $(12)$. This result is obtained by reexpressing the relative-c.m. ladder operators of (F10) in terms of single-particle coordinates and the oscillator length $b$ for the single-particle problem, via (F4), and then recognizing the relative-c.m. coordinates and momenta as defined in (12).

\section{Intrinsic ladder operators (many-body system)}

For the $A$-particle system (Sec. III C), we start from the oscillator creation operators $\mathbf{c}_{i}^{\dagger}$ $(i=1, \ldots, A)$ for the single-particle degrees of freedom,

$$
\mathbf{c}_{i}^{\dagger}=\frac{1}{\sqrt{2}}\left(b^{-1} \mathbf{x}_{i}-i b \mathbf{k}_{i}\right)
$$


Then intrinsic creation operators $\mathbf{c}_{i}^{\prime \dagger}$ are obtained by applying the substitutions $\mathbf{x}_{i} \rightarrow \mathbf{x}_{i}^{\prime}$ and $\mathbf{k}_{i} \rightarrow \mathbf{k}_{i}^{\prime}$ in (17), while the c.m. creation operator $\mathbf{c}_{\text {c.m. }}^{\dagger}$ is obtained from the sum of the single-particle creation operators:

$$
\mathbf{c}_{i}^{\prime \dagger}=\mathbf{c}_{i}^{\dagger}-\frac{1}{A} \sum_{j} \mathbf{c}_{j}^{\dagger} \quad \mathbf{c}_{\mathrm{c.m} .}^{\dagger}=\frac{1}{\sqrt{A}} \sum_{i} \mathbf{c}_{i}^{\dagger} .
$$

The definition of the c.m. coordinate and momentum in (17) gives the canonical commutatation relations for the c.m. degree of freedom, i.e., $\left[\mathbf{x}_{\mathrm{c.m} .}, \mathbf{k}_{\mathrm{c.m} .}\right]_{L_{0}}=-\sqrt{3} i \delta_{L_{0}, 0}$, with all others vanishing. Thus, the above definition for $\mathbf{c}_{\mathrm{c} . \mathrm{m}}^{\dagger}$ likewise yields canonical commutators for the ladder operators, i.e., $\left[\mathbf{c}_{\mathrm{c} . \mathrm{m} .}, \mathbf{c}_{\mathrm{c} . \mathrm{m} .}^{\dagger}\right]_{L_{0}}=-\sqrt{3} \delta_{L_{0}, 0}$, with all others vanishing. We may thus meaningfully define a c.m. number operator $N_{\text {c.m. }}=\mathbf{c}_{\text {c.m. }}^{\dagger} \cdot \mathbf{c}_{\text {c.m. }}$, with the usual properties for a number operator. However, the oscillator length $b_{\text {c.m. }}$ for the c.m. degree of freedom is not necessarily that associated with the single-particle oscillators. Rather, we identify

$$
\mathbf{c}_{\text {c.m. }}^{\dagger}=\frac{1}{\sqrt{2}}\left(b_{\text {c.m. }}{ }^{-1} \mathbf{x}_{\text {c.m. }}-i b_{\text {c.m. }} \mathbf{k}_{\text {c.m. } .}\right) \quad b_{\text {c.m. }}=\left\{\begin{array}{c}
1 / \sqrt{A} \\
1
\end{array}\right\} b,
$$

with the upper coefficient (in braces) applying under the mechanics convention and the lower coefficient applying under the symmetric convention for the many-body c.m. coordinate, as in (17). This result is obtained by reexpressing the c.m. ladder operator $\mathbf{c}_{\mathrm{c.m}}^{\dagger}$. of $(\mathrm{F} 13)$ in terms of single-particle coordinates and the oscillator length $b$ for the single-particle problem, via (F12), and then recognizing the c.m. coordinate $\mathbf{x}_{\mathrm{c} . \mathrm{m}}$. and momentum $\mathbf{k}_{\mathrm{c} . \mathrm{m}}$. defined in (17). The c.m. oscillator length $b_{\text {c.m. }}$ in (F14) defines the length scale for the zeropoint motion of the c.m. degree of freedom, which plays an important role in oscillator-basis calculations [115, e.g., when the explicit form of this zero-point motion must be used to apply corrections in NCCI calculations [139].

However, the overcomplete set of intrinsic coordinates and momenta defined by (17) do not obey canonical commutation relations. In fact, it may be verified that $\left[\mathbf{x}_{i}^{\prime}, \mathbf{k}_{j}^{\prime}\right]_{L_{0}}=$ $-\sqrt{3} i \delta_{L_{0}, 0}\left(\delta_{i j}-1 / A\right)$. Likewise, the intrinsic ladder operators do not obey canonical commutation relations, but, rather, have $\left[\mathbf{c}_{i}^{\prime}, \mathbf{c}_{j}^{\prime \dagger}\right]_{L_{0}}=-\sqrt{3} \delta_{L_{0}, 0}\left(\delta_{i j}-1 / A\right)$. This noncanonicality may be understood since the overcomplete set of ladder operators defined in $(\overline{F 13})$ do not provide a unitary change of basis on the bosonic creation operators $\mathbf{c}_{i}^{\dagger}$ defined in F12). Nonetheless, the intrinsic ladder operators do commute with the c.m. ladder operators, e.g., $\left[\mathbf{c}_{i}^{\prime}, \mathbf{c}_{\text {c.m. }}^{\dagger}\right]_{L_{0}}=0$. Moreover, it may be verified that the total number operator $N=\sum_{i} \mathbf{c}_{i}^{\dagger} \cdot \mathbf{c}_{i}$ separates into mutually-commuting intrinsic and c.m. contributions as $N=N^{\prime}+N_{\text {c.m. }}$, where $N^{\prime}=\sum_{i} \mathbf{c}_{i}^{\prime \dagger} \cdot \mathbf{c}_{i}^{\prime}$.

[1] Navrátil P, Barrett B R and Glöckle W 1999 Phys. Rev. C 59611

[2] Navrátil P, Quaglioni S, Stetcu I and Barrett B R 2009 J. Phys. G 36083101

[3] Barnea N and Novoselsky A 1997 Ann. Phys. (N.Y.) 256192

[4] Gartenhaus S and Schwartz C 1957 Phys. Rev. 108482

[5] Lipkin H J 1958 Phys. Rev. 1101395

[6] Seyfferth S 1967 Z. Phys. 200511

Seyfferth S 1967 Z. Phys. 204155

Seyfferth S 1967 Z. Phys. 207375 
[7] Eisenberg J M and Greiner W 1988 Nuclear Theory 3rd, revised ed vol 2 Excitation Mechanisms of the Nucleus (Amsterdam: North-Holland)

[8] Mosconi B and Ricci P 1987 Phys. Rev. C 3660

[9] Brussaard P J and Glaudemans P W M 1977 Shell-Model Applications in Nuclear Spectroscopy (Amsterdam: North-Holland Publishing Company)

[10] Glaudemans P W M 1985 A renewed look at light nuclei International Symposium on Nuclear Shell Models ed Vallieres M and Wildenthal B H (Philadelphia: World Scientific) p 2

[11] Bacca S, Barnea N and Schwenk A 2012 Phys. Rev. C 86034321

[12] Caprio M A, Maris P and Vary J P 2012 Phys. Rev. C 86034312

[13] Caprio M A, Maris P and Vary J P 2014 Phys. Rev. C 90034305

[14] Rosensteel G and Rowe D J 1977 Phys. Rev. Lett. 3810

[15] Rosensteel G and Rowe D J 1980 Ann. Phys. (N.Y.) 126343

[16] Rowe D J 1985 Rep. Prog. Phys. 481419

[17] Elliott J P 1958 Proc. R. Soc. London A 245128

Elliott J P 1958 Proc. R. Soc. London A 245562

Elliott J P and Harvey M 1963 Proc. R. Soc. London A 272557

Elliott J P and Wilsdon C E 1968 Proc. R. Soc. London A 302509

[18] Harvey M 1968 Adv. Nucl. Phys. 167

[19] Barrett B R, Navrátil P and Vary J P 2013 Prog. Part. Nucl. Phys. 69131

[20] McCoy A E 2018 Ab initio multi-irrep symplectic no-core configuration interaction calculations Ph.D. thesis University of Notre Dame URL https://curate.nd.edu/show/ pz50gt57p16

[21] McCoy A E, Caprio M A and Dytrych T 2018 Ann. Acad. Rom. Sci. Ser. Chem. Phys. Sci. 317 URL http://www.aos.ro/wp-content/anale/PCVol3Nr1Art.2.pdf

[22] McCoy A E, Caprio M A, Dytrych T and Fasano P J in press Phys. Rev. Lett.

[23] Eisenberg J M and Greiner W 1976 Nuclear Theory 2nd ed vol 3 Microscopic Theory of the Nucleus (Amsterdam: North-Holland)

[24] Kamuntavičius G P, Navrátil P, Barrett B R, Sapragonaite G and Kalinauskas R K 1999 Phys. Rev. C 60044304

[25] Gueorguiev V G, Navrátil P, Vary J P, Draayer J P and Pan F 2010 Nucl. Theory 29228

[26] Bethe H A and Rose M E 1937 Phys. Rev. 51283

[27] Brody T A, Jacob G and Moshinsky M 1960 Nucl. Phys. 1716

[28] Moshinsky M and Smirnov Y F 1996 The Harmonic Oscillator in Modern Physics (Amsterdam: Harwood Academic Publishers)

[29] Brink D M and Satchler G R 1994 Angular Momentum 3rd ed Oxford Science Publications (Oxford: Oxford University Press)

[30] Dytrych T, Sviratcheva K D, Draayer J P, Bahri C and Vary J P 2008 J. Phys. G 35123101

[31] Kretzschmar M 1960 Z. Phys. 158284

[32] Landau L D and Lifschitz E M 1981 Mechanics 3rd ed (Course of Theoretical Physics vol 1) (Oxford: Butterworth Heinemann) translated by J. B. Sykes and M. J. Kearsley

[33] McGrory J B and Wildenthal B H 1975 Phys. Lett. B 605

[34] Elliott J P and Skyrme T H R 1955 Proc. R. Soc. London A 232561

[35] Whitehead R R, Watt A, Cole B J and Morrison I 1977 Adv. Nucl. Phys. 9123

[36] Gloeckner D H and Lawson R D 1974 Phys. Lett. B 53313

[37] Lawson R D 1980 Theory of the Nuclear Shell Model (Oxford: Clarendon Press)

[38] Vary J P, Maris P, Fasano P J and Caprio M A 2018 JPS Conf. Proc. 23012001 
[39] Constantinou Ch, Caprio M A, Vary J P and Maris P 2017 Nucl. Sci. Techniques 28179

[40] Abe T, Maris P, Otsuka T, Shimizu N, Utsuno Y and Vary J P 2012 Phys. Rev. C 86054301

[41] Hagen G, Papenbrock T and Dean D J 2009 Phys. Rev. Lett. 103062503

Hagen G, Papenbrock T, Dean D J and Hjorth-Jensen M 2010 Phys. Rev. C 82034330

[42] Constantinou Ch 2017 Natural orbitals for the no-core configuration interaction approach Ph.D. thesis University of Notre Dame URL https://curate.nd.edu/show/ff365427x19

[43] Shull H and Löwdin P O 1955 J. Chem. Phys. 231362

[44] Weniger E J 1985 J. Math. Phys. 26276

[45] McCoy A E and Caprio M A 2016 J. Math. Phys. 57021708

[46] Wybourne B G 1974 Classical Groups for Physicists (New York: Wiley)

[47] O'Reilly M F 1982 J. Math. Phys. 232022

[48] Verhaar B J 1960 Nucl. Phys. 21508

[49] Hecht K T 1971 Nucl. Phys. A 17034

[50] Millener D J and Kurath D 1975 Nucl. Phys. A 255315

[51] Millener D J 1992 SU(3) in shell-model calculations Group Theory and Special Symmetries in Nuclear Physics ed Draayer J P and Jänecke J (Singapore: World Scientific) p 276

[52] Luo F Q, Caprio M A and Dytrych T 2013 Nucl. Phys. A 897109

[53] Dytrych T, Launey K D, Draayer J P, Maris P, Vary J P, Saule E, Catalyurek U, Sosonkina M, Langr D and Caprio M A 2013 Phys. Rev. Lett. 111252501

[54] Dytrych T, Maris P, Launey K D, Draayer J P, Vary J P, Langr D, Saule E, Caprio M A, Catalyurek U and Sosonkina M 2016 Comput. Phys. Commun. 207202

[55] Reske E J $1984 \mathrm{Sp}(6, R)$ symmetry and the giant quadrupole resonance of ${ }^{24} \mathrm{Mg} \mathrm{Ph} . \mathrm{D}$. thesis University of Michigan

[56] Suzuki Y and Hecht K T 1986 Nucl. Phys. A 455315

[57] Suzuki Y 1986 Nucl. Phys. A 448395

[58] Cockrell C, Vary J P and Maris P 2012 Phys. Rev. C 86034325

[59] Negele J W and Orland H 1988 Quantum Many-Particle Systems (Redwood City, CA: Addison-Wesley)

[60] Varshalovich D A, Moskalev A N and Khersonskii V K 1988 Quantum Theory of Angular Momentum (Singapore: World Scientific)

[61] Friar J L, Martorell J and Sprung D W L 1997 Phys. Rev. A 564579

[62] Church E L and Weneser J 1956 Phys. Rev. 1031035

[63] Bohr A and Mottelson B R 1998 Nuclear Structure vol 1 Single-Particle Motion (Singapore: World Scientific)

[64] Ring P and Schuck P 1980 The Nuclear Many-Body Problem (New York: Springer-Verlag)

[65] Eisenberg J M and Greiner W 1987 Nuclear Theory 3rd ed vol 1 Nuclear Models: Collective and Single-Particle Phenomena (Amsterdam: North-Holland)

[66] Suhonen J 2007 From Nucleons to Nucleus (Berlin: Springer-Verlag)

[67] Bernstein A, Brown V and Madsen V 1981 Phys. Lett. B 103255

[68] Rowe D J 2010 Nuclear Collective Motion: Models and Theory (Singapore: World Scientific)

[69] Rowe D J and Wood J L 2010 Fundamentals of Nuclear Models: Foundational Models (Singapore: World Scientific)

[70] Rosensteel G $1992 \mathrm{Sp}(3, \mathbb{R})$ tensors in nuclear physics Group Theory and Special Symmetries in Nuclear Physics ed Draayer J P and Jänecke J (Singapore: World Scientific) p 332

[71] Escher J and Leviatan A 2002 Phys. Rev. C 65054309

[72] Draayer J P and Akiyama Y 1973 J. Math. Phys. 141904 
[73] Escher J and Draayer J P 1998 J. Math. Phys. 395123

[74] Henley E M 1969 Charge independence and charge symmetry of nuclear forces Isospin in Nuclear Physics ed Wilkinson D H (Amsterdam: North-Holland) p 15

[75] Rose M E 1957 Elementary Theory of Angular Momentum (New York: Wiley)

[76] Edmonds A R 1960 Angular Momentum in Quantum Mechanics 2nd ed (Investigations in Physics no 4) (Princeton, New Jersey: Princeton University Press)

[77] French J B 1966 Multipole and sum-rule methods in spectroscopy Proceedings of the International School of Physics "Enrico Fermi", Course XXXVI ed Bloch C (New York: Academic Press) p 278

[78] Chen J Q, Chen B Q and Klein A 1993 Nucl. Phys. A 55461

[79] Caprio M A, Skrabacz J H and Iachello F 2011 J. Phys. A 44075303

[80] Iachello F 2015 Lie Algebras and Applications 2nd ed (Lecture Notes in Physics vol 891) (Berlin: Springer)

[81] Caprio M A 2005 J. Phys. A 386385

[82] Navrátil P, Barrett B R and Glöckle W 1999 Phys. Rev. C 59611

[83] Navrátil P, Quaglioni S, Stetcu I and Barrett B R 2009 J. Phys. G 36083101

[84] Barnea N and Novoselsky A 1997 Ann. Phys. (N.Y.) 256192

[85] Gartenhaus S and Schwartz C 1957 Phys. Rev. 108482

[86] Lipkin H J 1958 Phys. Rev. 1101395

[87] Seyfferth S 1967 Z. Phys. 200511

Seyfferth S 1967 Z. Phys. 204155

Seyfferth S 1967 Z. Phys. 207375

[88] Eisenberg J M and Greiner W 1988 Nuclear Theory 3rd, revised ed vol 2 Excitation Mechanisms of the Nucleus (Amsterdam: North-Holland)

[89] Mosconi B and Ricci P 1987 Phys. Rev. C 3660

[90] Brussaard P J and Glaudemans P W M 1977 Shell-Model Applications in Nuclear Spectroscopy (Amsterdam: North-Holland Publishing Company)

[91] Glaudemans P W M 1985 A renewed look at light nuclei International Symposium on Nuclear Shell Models ed Vallieres M and Wildenthal B H (Philadelphia: World Scientific) p 2

[92] Bacca S, Barnea N and Schwenk A 2012 Phys. Rev. C 86034321

[93] Caprio M A, Maris P and Vary J P 2012 Phys. Rev. C 86034312

[94] Caprio M A, Maris P and Vary J P 2014 Phys. Rev. C 90034305

[95] Rosensteel G and Rowe D J 1977 Phys. Rev. Lett. 3810

[96] Rosensteel G and Rowe D J 1980 Ann. Phys. (N.Y.) 126343

[97] Rowe D J 1985 Rep. Prog. Phys. 481419

[98] Elliott J P 1958 Proc. R. Soc. London A 245128

Elliott J P 1958 Proc. R. Soc. London A 245562

Elliott J P and Harvey M 1963 Proc. R. Soc. London A 272557

Elliott J P and Wilsdon C E 1968 Proc. R. Soc. London A 302509

[99] Harvey M 1968 Adv. Nucl. Phys. 167

[100] Barrett B R, Navrátil P and Vary J P 2013 Prog. Part. Nucl. Phys. 69131

[101] McCoy A E 2018 Ab initio multi-irrep symplectic no-core configuration interaction calculations Ph.D. thesis University of Notre Dame URL https://curate.nd.edu/show/ pz50gt57p16

[102] McCoy A E, Caprio M A and Dytrych T 2018 Ann. Acad. Rom. Sci. Ser. Chem. Phys. Sci. 317 URL http://www . aos.ro/wp-content/anale/PCVol3Nr1Art.2.pdf 
[103] McCoy A E, Caprio M A, Dytrych T and Fasano P J in press Phys. Rev. Lett.

[104] Eisenberg J M and Greiner W 1976 Nuclear Theory 2nd ed vol 3 Microscopic Theory of the Nucleus (Amsterdam: North-Holland)

[105] Kamuntavičius G P, Navrátil P, Barrett B R, Sapragonaite G and Kalinauskas R K 1999 Phys. Rev. C 60044304

[106] Gueorguiev V G, Navrátil P, Vary J P, Draayer J P and Pan F 2010 Nucl. Theory 29228

[107] Bethe H A and Rose M E 1937 Phys. Rev. 51283

[108] Brody T A, Jacob G and Moshinsky M 1960 Nucl. Phys. 1716

[109] Moshinsky M and Smirnov Y F 1996 The Harmonic Oscillator in Modern Physics (Amsterdam: Harwood Academic Publishers)

[110] Brink D M and Satchler G R 1994 Angular Momentum 3rd ed Oxford Science Publications (Oxford: Oxford University Press)

[111] Dytrych T, Sviratcheva K D, Draayer J P, Bahri C and Vary J P 2008 J. Phys. G 35123101

[112] Kretzschmar M 1960 Z. Phys. 158284

[113] Landau L D and Lifschitz E M 1981 Mechanics 3rd ed (Course of Theoretical Physics vol 1) (Oxford: Butterworth Heinemann) translated by J. B. Sykes and M. J. Kearsley

[114] McGrory J B and Wildenthal B H 1975 Phys. Lett. B 605

[115] Elliott J P and Skyrme T H R 1955 Proc. R. Soc. London A 232561

[116] Whitehead R R, Watt A, Cole B J and Morrison I 1977 Adv. Nucl. Phys. 9123

[117] Gloeckner D H and Lawson R D 1974 Phys. Lett. B 53313

[118] Lawson R D 1980 Theory of the Nuclear Shell Model (Oxford: Clarendon Press)

[119] Vary J P, Maris P, Fasano P J and Caprio M A 2018 JPS Conf. Proc. 23012001

[120] Constantinou Ch, Caprio M A, Vary J P and Maris P 2017 Nucl. Sci. Techniques 28179

[121] Abe T, Maris P, Otsuka T, Shimizu N, Utsuno Y and Vary J P 2012 Phys. Rev. C 86054301

[122] Hagen G, Papenbrock T and Dean D J 2009 Phys. Rev. Lett. 103062503

Hagen G, Papenbrock T, Dean D J and Hjorth-Jensen M 2010 Phys. Rev. C 82034330

[123] Constantinou Ch 2017 Natural orbitals for the no-core configuration interaction approach Ph.D. thesis University of Notre Dame URL https://curate.nd.edu/show/ff365427x19

[124] Shull H and Löwdin P O 1955 J. Chem. Phys. 231362

[125] Weniger E J 1985 J. Math. Phys. 26276

[126] McCoy A E and Caprio M A 2016 J. Math. Phys. 57021708

[127] Wybourne B G 1974 Classical Groups for Physicists (New York: Wiley)

[128] O'Reilly M F 1982 J. Math. Phys. 232022

[129] Verhaar B J 1960 Nucl. Phys. 21508

[130] Hecht K T 1971 Nucl. Phys. A 17034

[131] Millener D J and Kurath D 1975 Nucl. Phys. A 255315

[132] Millener D J 1992 SU(3) in shell-model calculations Group Theory and Special Symmetries in Nuclear Physics ed Draayer J P and Jänecke J (Singapore: World Scientific) p 276

[133] Luo F Q, Caprio M A and Dytrych T 2013 Nucl. Phys. A 897109

[134] Dytrych T, Launey K D, Draayer J P, Maris P, Vary J P, Saule E, Catalyurek U, Sosonkina M, Langr D and Caprio M A 2013 Phys. Rev. Lett. 111252501

[135] Dytrych T, Maris P, Launey K D, Draayer J P, Vary J P, Langr D, Saule E, Caprio M A, Catalyurek U and Sosonkina M 2016 Comput. Phys. Commun. 207202

[136] Reske E J $1984 \mathrm{Sp}(6, R)$ symmetry and the giant quadrupole resonance of ${ }^{24} \mathrm{Mg} \mathrm{Ph} . \mathrm{D}$. thesis University of Michigan

[137] Suzuki Y and Hecht K T 1986 Nucl. Phys. A 455315 
[138] Suzuki Y 1986 Nucl. Phys. A 448395

[139] Cockrell C, Vary J P and Maris P 2012 Phys. Rev. C 86034325

[140] Negele J W and Orland H 1988 Quantum Many-Particle Systems (Redwood City, CA: Addison-Wesley)

[141] Varshalovich D A, Moskalev A N and Khersonskii V K 1988 Quantum Theory of Angular Momentum (Singapore: World Scientific)

[142] Friar J L, Martorell J and Sprung D W L 1997 Phys. Rev. A 564579

[143] Church E L and Weneser J 1956 Phys. Rev. 1031035

[144] Bohr A and Mottelson B R 1998 Nuclear Structure vol 1 Single-Particle Motion (Singapore: World Scientific)

[145] Ring P and Schuck P 1980 The Nuclear Many-Body Problem (New York: Springer-Verlag)

[146] Eisenberg J M and Greiner W 1987 Nuclear Theory 3rd ed vol 1 Nuclear Models: Collective and Single-Particle Phenomena (Amsterdam: North-Holland)

[147] Suhonen J 2007 From Nucleons to Nucleus (Berlin: Springer-Verlag)

[148] Bernstein A, Brown V and Madsen V 1981 Phys. Lett. B 103255

[149] Rowe D J 2010 Nuclear Collective Motion: Models and Theory (Singapore: World Scientific)

[150] Rowe D J and Wood J L 2010 Fundamentals of Nuclear Models: Foundational Models (Singapore: World Scientific)

[151] Rosensteel G $1992 \mathrm{Sp}(3, \mathbb{R})$ tensors in nuclear physics Group Theory and Special Symmetries in Nuclear Physics ed Draayer J P and Jänecke J (Singapore: World Scientific) p 332

[152] Escher J and Leviatan A 2002 Phys. Rev. C 65054309

[153] Draayer J P and Akiyama Y 1973 J. Math. Phys. 141904

[154] Escher J and Draayer J P 1998 J. Math. Phys. 395123

[155] Henley E M 1969 Charge independence and charge symmetry of nuclear forces Isospin in Nuclear Physics ed Wilkinson D H (Amsterdam: North-Holland) p 15

[156] Rose M E 1957 Elementary Theory of Angular Momentum (New York: Wiley)

[157] Edmonds A R 1960 Angular Momentum in Quantum Mechanics 2nd ed (Investigations in Physics no 4) (Princeton, New Jersey: Princeton University Press)

[158] French J B 1966 Multipole and sum-rule methods in spectroscopy Proceedings of the International School of Physics "Enrico Fermi", Course XXXVI ed Bloch C (New York: Academic Press) p 278

[159] Chen J Q, Chen B Q and Klein A 1993 Nucl. Phys. A 55461

[160] Caprio M A, Skrabacz J H and Iachello F 2011 J. Phys. A 44075303

[161] Iachello F 2015 Lie Algebras and Applications 2nd ed (Lecture Notes in Physics vol 891) (Berlin: Springer)

[162] Caprio M A 2005 J. Phys. A 386385 